\title{
Minimal controllability time for the heat equation under unilateral state or control constraints
}

\author{
Jérôme Lohéac* Emmanuel Trélat ${ }^{\dagger} \quad$ Enrique Zuazua $\mathbb{1}$
}

February 6, 2017

\begin{abstract}
The heat equation with homogeneous Dirichlet boundary conditions is well known to preserve nonnegativity. Besides, due to infinite velocity propagation, the heat equation is nullcontrollable within arbitrary small time, with controls supported in any arbitrarily open subset of the domain (or its boundary) where heat diffuses.

The following question then arises naturally: can the heat dynamics be controlled from a positive initial steady-state to a positive final one, requiring that the state remains nonnegative along the controlled time-dependent trajectory? We show that this state-constrained controllability property can be achieved if the control time is large enough, but that it fails to be true in general if the control time is too short, thus showing the existence of a positive minimal controllability time. In other words, in spite of infinite velocity propagation, realizing controllability under the unilateral nonnegativity state constraint requires a positive minimal time.

We establish similar results for unilateral control constraints.

We give some explicit bounds on the minimal controllability time, first in $1 \mathrm{D}$ by using the sinusoidal spectral expansion of solutions, and then in multi-D on any bounded domain. We illustrate our results with numerical simulations, and we discuss similar issues for other control problems with various boundary conditions.
\end{abstract}

\section{Contents}

1 Introduction

2 Minimal time for the Dirichlet controlled 1D heat equation under nonnegativity constraints

2.1 Main results . . . . . . . . . . . . . . . . . . . . 5

2.2 Weak solutions with controls that are Radon measures $\ldots \ldots \ldots \ldots$

2.3 Proof of Theorem $1 \ldots \ldots \ldots \ldots$

${ }^{*}$ LUNAM Université, IRCCyN UMR CNRS 6597 (Institut de Recherche en Communications et Cybernétique de Nantes), École des Mines de Nantes, 4 rue Alfred Kastler, 44307 Nantes, France (jerome.loheac@irccyn.ec-nantes.fr).

${ }^{\dagger}$ Sorbonne Universités, UPMC Univ Paris 06, CNRS UMR 7598, Laboratoire Jacques-Louis Lions, F-75005, Paris, France (emmanuel.trelat@upmc.fr).

${ }^{\ddagger}$ DeustoTech, Fundación Deusto, Avda Universidades, 24, 48007, Bilbao - Basque Country - Spain (enrique.zuazua@deusto.es)

${ }^{\S}$ Departamento de Matemáticas, Universidad Autónoma de Madrid, Cantoblanco, 28049 Madrid - Spain

IFacultad Ingeniería, Universidad de Deusto, Avda. Universidades, 24, 48007, - Basque Country - Spain

"Sorbonne Universités, UPMC Univ Paris 06, CNRS UMR 7598, Laboratoire Jacques-Louis Lions, F-75005, Paris, France. 
2.4 Proof of Proposition $1 \ldots \ldots \ldots \ldots \ldots$

2.5 Proof of Theorem $2 \ldots \ldots \ldots \ldots \ldots \ldots \ldots \ldots$

2.6 Lower estimates of the minimal time for a constant initial datum . . . . . . . . 12

3 Minimal time for Dirichlet controlled multi-D heat equations under nonnegativity constraints

3.1 Heat equation in a ball with nonnegative Dirichlet controls . . . . . . . . . . . 14

3.2 General domain and nonnegativity state constraint . . . . . . . . . . . . 18

3.3 Relationship with the viscous Hamilton-Jacobi equation . . . . . . . . . . 20

4 Generalizations $\quad \mathbf{2 0}$

4.1 Mixed Dirichlet-Neumann boundary conditions . . . . . . . . . . . . . 20

4.2 Neumann control under nonnegativity state constraints . . . . . . . . . . . . 21

4.3 Internal control with Neumann boundary conditions . . . . . . . . . . . . . . 24

4.4 Neumann boundary control with control constraints in 1D . . . . . . . . . . 25

5 Numerical simulations $\quad \mathbf{2 7}$

5.1 Dirichlet boundary controls with nonnegativity control constraints . . . . . . 27

5.2 Neumann controls and state constraints . . . . . . . . . . . . . . 33

5.3 Convergence result for the minimal time . . . . . . . . . . . . . 35

6 Conclusion, open problems and perspectives

\section{Introduction}

This work is devoted to analyze the controllability properties on the heat equation under natural unilateral constraints on the state. The free heat equation, in the absence of control and, for instance, for homogeneous Dirichlet boundary conditions, is well known to preserve nonnegativity. Besides, due to infinite velocity propagation, the heat equation is null-controllable within arbitrary small time with controls acting on an arbitrarily small open subset of the domain (of its boundary) where heat propagates.

The following question then arises naturally: if the initial datum and the final target are positive steady-states, can the heat dynamics be controlled under a nonnegativity state constraint along the whole trajectory?

In this paper, we present two types of complementary results exhibiting a "waiting or minimal time phenomenon". Roughly, we show that, given positive initial and final steadystates, the system can be controlled while preserving nonnegativity of the state provided that the time horizon $[0, T]$ be long enough. This is not surprising: in fact, when the time-interval is long, we expect the control property to be achieved with controls of small amplitude, thus ensuring small deformations of the state and, in particular, preserving its positivity. As we will see, it suffices that the target be positive, regardless of the sign of the initial datum, to ensure that the controls are positive as well. If, in addition, the initial datum is positive as well, the positivity of the control ensures positivity of the state everywhere.

Maybe more surprisingly, we also prove that, if the time-interval is too short, then the controllability property fails under the nonnegativity state-constraint. In other words, even if the initial datum and final steady target are positive, nonnegativity cannot be preserved along the controlled trajectory if the time horizon is too short: in spite of infinite velocity propagation, controlling the system then requires to violate the natural nonnegativity constraint on the state. This means, roughly speaking, that the necessary action of the control to avoid the state to cross the limit established by the constraint, is an impediment for the state to reach the target, unless the control time horizon is long enough.

This negative result, which is counterintuitive to some extent, is a serious warning for the practical use of existing controllability results, that are valid within arbitrarily short time since, as often in applications, state-constraints need to be preserved along controlled trajectories. 
This is the case, for instance, when the heat equation or, more generally, the diffusive system under consideration, models the propagation of a population density, as in the context of population dynamics.

We mainly focus on the heat equation with Dirichlet boundary control. To simplify the presentation, we first analyze the 1D heat equation although, as we shall see, the results can then be extended to the multi-D case.

To begin with, we consider the 1D heat equation with Dirichlet boundary controls:

$$
\begin{aligned}
\partial_{t} y(t, x) & =\partial_{x}^{2} y(t, x) & & (t>0, x \in(0,1)), \\
y(t, 0) & =u_{0}(t) & & (t>0), \\
y(t, 1) & =u_{1}(t) & & (t>0),
\end{aligned}
$$

with constant initial condition $y(0, x)=\mathrm{y}^{0}>0$ (steady-state). The controls are represented by the time-dependent functions $u_{0}(t)$ and $u_{1}(t)$ that act on the system at the boundary points $x=0$ and $x=1$, respectively. Here, we assume that the initial state is a positive constant $\mathrm{y}^{0}>0$, thus a steady-state of the system that can be sustained with constant controls $u_{0}(t) \equiv u_{1}(t) \equiv \mathrm{y}^{0}$.

Given a constant steady-state target $\mathrm{y}^{1}>0$ (steady-state sustainable with the constant controls $\left.u_{0}(t) \equiv u_{1}(t) \equiv \mathrm{y}^{1}\right)$, as mentioned above, whatever the time of control $T>0$ is, we know that there exist controls $u_{0}$ and $u_{1}$ in $L^{2}(0, T)$ steering the system to $\mathrm{y}^{1}$ in time $T$, i.e., such that

$$
y(T, x)=\mathrm{y}^{1} \quad(x \in[0,1] \text { a.e. }) .
$$

The problem we analyze is whether the controls $u_{0}$ and $u_{1}$ can be chosen so that the solution remains nonnegative along the time interval, i.e., whether the following unilateral state constraint can be satisfied:

$$
y(t, x) \geqslant 0, \quad \forall t \in(0, T), x \in(0,1) .
$$

Of course we are only interested in the nontrivial case where $\mathrm{y}^{1} \neq \mathrm{y}^{0}$. Otherwise, when $\mathrm{y}^{1}=\mathrm{y}^{0}$ the trivial trajectory $y \equiv \mathrm{y}^{0}=\mathrm{y}^{1}$ solves the problem with constant controls $u_{0} \equiv u_{1} \equiv \mathrm{y}^{0}=\mathrm{y}^{1}$. In this paper, we establish two types of results:

- Controllability can be achieved while preserving nonnegativity (3) if $T>0$ is large enough;

- Controllability fails if $T>0$ is too small; more precisely, there exists $\underline{T}\left(\mathrm{y}^{0}, \mathrm{y}^{1}\right)>0$ such that, for every $T \in\left(0, \underline{T}\left(\mathrm{y}^{0}, \mathrm{y}^{1}\right)\right)$, there do not exist any controls $u_{0}$ and $u_{1}$ such that the solution $y$ of the heat equation satisfies (2) while satisfying the state constraint (3). This means that there is a positive minimal time, or waiting time.

In the absence of state-constraints, it is well known that, given any $T>0$, there exist controls such that the solution $y$ of the heat equation reaches $\mathrm{y}^{1}$ in time $T$ (see for instance $[25, \S 11.5]$ and see references therein), and this result is even valid for much more general heat equations with various types of controls and boundary conditions in multi-D. But it is important to emphasize that usual results of the literature do not take into consideration the objective of preserving state-constraints, and actually the numerical results that one can find in the existing literature show that the corresponding controls and controlled trajectories enjoy significant oscillations (see [16]), thus showing the difficulties that may arise under stateconstraints.

Our result ensuring that constrained controllability can be achieved if $T$ is large enough can be roughly proved as follows. When $T$ is large, the cost of control tends to zero. In fact in the present setting of Dirichlet boundary controls it can be easily proved that the cost of control can be made exponentially small as $T \rightarrow+\infty$, and that for $T$ large enough the control can be made arbitrarily small in $L^{\infty}$ norm. Moreover, the comparison or maximum principle for the heat equation guarantees that the controlled trajectory remains in a tubular neighborhood of $\mathrm{y}^{0}$ and $\mathrm{y}^{1}$ all along the time horizon $[0, T]$ and, in particular, preserves the nonnegativity constraint when $\mathrm{y}^{0}$ and $\mathrm{y}^{1}$ are positive. Note that this argument fails in short time when the size of controls may become large. 
To prove that, if the control time $T$ is too small, then nonnegativity of the solution cannot be guaranteed in general (thus, there is a positive "minimal" or "waiting time"), we use the classical maximum or comparison principle for the heat equation. Since $\mathrm{y}^{0}>0$, controllability under the state constraint (3) is in fact equivalent to the apparently weaker property

$$
u_{0}(t)=y(t, 0) \geqslant 0 \quad \text { and } \quad u_{1}(t)=y(t, 1) \geqslant 0 \quad(t \in(0, T) \text { a.e. }) .
$$

Indeed, when the nonnegativity state constraint (3) is satisfied, the boundary constraint (4) is satisfied too. On the other hand, the latter and the positivity of the initial state suffice to show that the state is nonnegative everywhere. This shows the equivalence between the problems of control under nonnegative state and control constraints, for the heat equation with Dirichlet controls.

In view of this, in some particular cases, it is easy to see that a positive minimal time is required for controlling the system under nonnegativity state constraints. This occurs if the final target is smaller than the initial datum, i.e., if $0<\mathrm{y}^{1}<\mathrm{y}^{0}$ (recall that $\mathrm{y}^{0}$ and $\mathrm{y}^{1}$ are constant). Indeed, we infer from the maximum or comparison principle for the heat equation that, whatever the controls $u_{0}$ and $u_{1}$ are, if the solution $y$ satisfies the nonnegativity state constraint (3), then, due to (4), we must have

$$
y(t, x) \geqslant \tilde{y}(t, x) \quad(t \in(0, T), x \in(0,1) \text { a.e. }),
$$

where $\tilde{y}$ is solution of

$$
\begin{aligned}
\partial_{t} \tilde{y}(t, x) & =\partial_{x}^{2} \tilde{y}(t, x) & & (t>0, x \in(0,1)), \\
\tilde{y}(t, 0) & =\tilde{y}(t, 1)=0 & & (t>0), \\
\tilde{y}(0, x) & =\mathrm{y}^{0} & & (x \in(0,1)),
\end{aligned}
$$

and then, since $\tilde{y}(0, x) \geqslant \mathrm{y}^{0} \sin (\pi x)$, we infer that

$$
\tilde{y}(t, x) \geqslant \mathrm{y}^{0} \exp \left(-\pi^{2} t\right) \sin (\pi x),
$$

$\sin (\pi \cdot)$ being the first eigenfunction of the Dirichlet-Laplacian in $(0,1)$. It follows that

$$
\sup _{x \in[0,1]} y(t, x) \geqslant \mathrm{y}^{0} \exp \left(-\pi^{2} t\right),
$$

and thus, if $0<\mathrm{y}^{1}<\mathrm{y}^{0}$, then there exists a positive minimal time $\underline{T}\left(\mathrm{y}^{0}, \mathrm{y}^{1}\right)>0$ to steer the system from $\mathrm{y}^{0}$ to $\mathrm{y}^{1}$, and we have

$$
\underline{T}\left(\mathrm{y}^{0}, \mathrm{y}^{1}\right) \geqslant \frac{1}{\pi^{2}} \ln \left(\frac{\mathrm{y}^{0}}{\mathrm{y}^{1}}\right) .
$$

In the present paper, we will also establish that a similar but much less obvious phenomenon occurs when $y^{1}>y^{0}>0$. This fact, contrarily to the previous observation corresponding to the case where $0<\mathrm{y}^{1}<\mathrm{y}^{0}$, is quite surprising because, at the first view, nothing prevents $y$ from increasing as fast as needed under the action of nonnegative controls. To handle this case, we will need to study more deeply the Dirichlet control problem, using spectral expansions of solutions. This will be done in Section 2, where moreover the initial datum $\mathrm{y}^{0}$ can be any function in $L^{2}(0,1)$.

In Section 3, we extend these results to the multi-dimensional case. As mentioned above, the fact that constrained controllability can be achieved in long time intervals is a consequence of classical results on the decay of the control cost as $T \rightarrow+\infty$, and thus it is easy to extend to the multi-D setting. In contrast, the $1 \mathrm{D}$ proof of the necessity of a waiting time uses explicit properties of the 1D Laplacian. We first extend this result to the multi-D ball, by using the explicit form of radially symmetric eigenfunctions and getting explicit lower bounds on the waiting time. Once this is done, the result is extended to any other domain by comparison with the largest ball included in the domain. Note that, in Section 3.3, we present an interesting 
remark done by M. Tucsnak [24], indicating that the waiting time phenomenon for the control of the linear heat equation under nonnegativity state-constraints is related to that of [17] on the control of the viscous Hamilton-Jacobi equations.

In Section 4, we then consider similar issues in various other situations. First, in the case of Neumann boundary control, the same arguments lead to a waiting time principle under stateconstraints. The reason is the same: if the state remains positive, it can always be viewed as a controlled trajectory with positive Dirichlet controls. But we also consider, among others, the case of interior controls applied in a subdomain of the region where heat diffuses.

In Section 5, we present some numerical simulations that confirm our analytical results, shedding new light on the nature of the constrained controls in the minimal control time and raising new questions.

We conclude with a section devoted to open problems and some possible extensions.

\section{Minimal time for the Dirichlet controlled 1D heat equation under nonnegativity constraints}

In this section, we consider the 1D heat equation (1) with Dirichlet boundary controls. We assume that the final target $\mathrm{y}^{1}$ is a positive constant, which corresponds to a steady-state of the system. More generally than in the previous section, the initial datum $\mathrm{y}^{0}$ can now be any element of $L^{2}(0,1)$.

We have seen in Section 1 that, if $\mathrm{y}^{0}>0$, then controllability under the nonnegativity state constraint (3) is equivalent to controllability under the nonnegativity control constraints (4).

This is why, in this section, we consider unilateral nonnegativity control constraints. But all results hereafter are then valid as well for unilateral nonnegativity state constraints.

\subsection{Main results}

We consider an arbitrary $\mathrm{y}^{0} \in L^{2}(0,1)$ (without any sign requirement), and we consider the problem of steering the heat equation (1) from $\mathrm{y}^{0}$ to the positive steady-state $\mathrm{y}^{1}$, under the unilateral control constraints

$$
u_{0}(t) \geqslant 0, \quad u_{1}(t) \geqslant 0, \quad \text { a.e. } t \in(0, T)
$$

Our first main result states the existence of such controls for a large enough time $T$.

Theorem 1. Let $\mathrm{y}^{0} \in L^{2}(0,1)$ be arbitrary, and let $\mathrm{y}^{1}>0$ be a positive constant, such that $\mathrm{y}^{0} \neq \mathrm{y}^{1}$. There exist $T>0$, depending on the initial and final data $\mathrm{y}^{0}, \mathrm{y}^{1}$, and controls $u_{0} \in L^{1}(0, T)$ and $u_{1} \in L^{1}(0, T)$, satisfying the nonnegativity constraints (5), such that the corresponding solution $y$ of $(1)$, with $y(0)=\mathrm{y}^{0}$, satisfies $y(T)=\mathrm{y}^{1}$.

Theorem 1 is proved in Section 2.3. Note that, as we will see in the proof, for $T>0$ large enough the controls are actually positive (and $\mathrm{y}^{0}$ need not be nonnegative), due to positivity of the target at the final time.

Remark 1. We can actually state the following result of controllability to trajectories, slightly extending Theorem 1:

Let $\overline{\mathrm{y}}^{0} \in L^{2}(0,1)$ and let $\bar{u}_{0}$ and $\bar{u}_{1}$ in $L_{\text {loc }}^{1}([0,+\infty))$ be arbitrary. Let $\bar{y}$ be the solution of (1) corresponding to the initial condition $\bar{y}(0)=\overline{\mathrm{y}}^{0}$ and to the controls $\bar{u}_{0}$ and $\bar{u}_{1}$. We assume that there exists $\nu>0$ such that $\bar{u}_{i}(t) \geqslant \nu$, for $i=0,1$ and for almost every $t \in[0,+\infty)$.

Then, for every $\mathrm{y}^{0} \in L^{2}(0,1)$, there exist $T>0$ and nonnegative controls $u_{0} \epsilon$ $L^{1}(0, T)$ and $u_{1} \in L^{1}(0, T)$ such that the solution $y$ of (1) corresponding to the initial condition $y(0)=\mathrm{y}^{0}$ and to the controls $u_{0}$ and $u_{1}$ satisfies $y(T)=\bar{y}(T)$. 
This result is an extension of Theorem 1 (where $\bar{y} \equiv \mathrm{y}^{1}$ and $\bar{u}_{0}=\bar{u}_{1}=0$ ). The proof is similar to the one of Theorem 1 , by subtracting $\bar{y}$ to $y$ so we have to steer $\mathrm{y}^{0}-\overline{\mathrm{y}}^{0}$ to 0 with control $v=u-\bar{u}$ and we prove that there exists a control $v \in H^{1}(0, T)$ (for $T$ large enough) satisfying $v(t) \geqslant-\nu$, i.e., $u(t) \geqslant 0$.

Let us now study the minimal time required to steer $\mathrm{y}^{0}$ to $\mathrm{y}^{1}$ in Theorem 1 , and prove that it is positive. We define the minimal controllability time

$$
\underline{T}\left(\mathrm{y}^{0}, \mathrm{y}^{1}\right)=\inf \left\{T>0, \exists u_{0}, u_{1} \in L^{1}(0, T) \text { s.t. } u_{0} \geqslant 0, u_{1} \geqslant 0 \text { and } y(0)=\mathrm{y}^{0}, y(T)=\mathrm{y}^{1}\right\} .
$$

It is defined here with controls in $L^{1}(0, T)$, but it could also be defined with other functional control spaces. However, on the one part, the arguments we shall develop, based on spectral expansions, lead to bounds in $L^{1}(0, T)$ that are uniform with respect to $T>T\left(\mathrm{y}^{0}, \mathrm{y}^{1}\right)$ and, consequently, are valid in the space of measures for the minimal time $\underline{T}\left(\mathrm{y}^{0}, \mathrm{y}^{1}\right)$. On the other part, the following result shows that, if controllability can be achieved in time $T$ with controls in $L^{1}(0, T)$, then it can also be achieved in time $T+\tau$ with $\tau>0$ arbitrarily small, with controls that are arbitrarily more regular.

Proposition 1. Let $\mathrm{y}^{0} \in L^{2}(0,1)$ be arbitrary, and let $\mathrm{y}^{1}>0$ be a positive constant. Let $T>0$ be such that there exist controls $u_{0} \in L^{1}(0, T)$ and $u_{1} \in L^{1}(0, T)$, satisfying the nonnegativity constraints (5), for which the corresponding solution $y$ of $(1)$, with $y(0)=\mathrm{y}^{0}$, satisfies $y(T)=$ $\mathrm{y}^{1}$.

Now, let $n \in \mathbb{N}^{*}$ and let $\tau>0$ be arbitrary. Then there exist controls $\tilde{u}_{0} \in C^{n}([0, T+\tau])$ and $\tilde{u}_{1} \in C^{n}([0, T+\tau])$, satisfying the nonnegativity constraints (5), such that the corresponding solution $\tilde{y}$ of $(1)$, with $\tilde{y}(0)=\mathrm{y}^{0}$, satisfies $\tilde{y}(T+\tau)=\mathrm{y}^{1}$.

This result will be proved in Section 2.4.

Our second main result is the following.

Theorem 2. Given any $\mathrm{y}^{0} \in L^{2}(0,1)$ and any positive constant $\mathrm{y}^{1}>0$ such that $\mathrm{y}^{0} \neq \mathrm{y}^{1}$, we have $\underline{T}\left(\mathrm{y}^{0}, \mathrm{y}^{1}\right)>0$.

Moreover, there exist nonnegative controls $\underline{u}_{0}, \underline{u}_{1} \in \mathcal{M}\left(0, \underline{T}\left(\mathrm{y}^{0}, \mathrm{y}^{1}\right)\right)$ (the space of Radon measures) steering the heat equation (1) from $\mathrm{y}^{0}$ to $\mathrm{y}^{1}$ in time $\underline{T}\left(\mathrm{y}^{0}, \mathrm{y}^{1}\right)$.

This result states not only that the minimal time required to steer $\mathrm{y}^{0}$ to $\mathrm{y}^{1}$ in Theorem 1 is positive, but also that controllability exactly at the minimal time $\underline{T}\left(\mathrm{y}^{0}, \mathrm{y}^{1}\right)$ can be achieved with controls that are Radon measures. We do not know whether they can be taken more regular or not. When controls are Radon measures, the concept of weak solution of the heat equation (defined by transposition) is recalled in Section 2.2.

Remark 2. Given any $T>\underline{T}\left(\mathrm{y}^{0}, \mathrm{y}^{1}\right)$, by definition there exist controls $u_{0}$ and $u_{1}$ in $L^{1}(0, T)$, satisfying the nonnegativity constraints (5), steering the heat equation (1) from $\mathrm{y}^{0}$ to $\mathrm{y}^{1}$ in time $T$. We claim that, if $\mathrm{y}^{0}$ is symmetric with respect to $x=1 / 2$, meaning that $\mathrm{y}^{0}(x)=\mathrm{y}^{0}(1-x)$ for almost every $x \in[0,1]$, then we can take $u_{0}=u_{1}$.

Remark 3. As we will see in the proof, the fact that $\underline{T}\left(\mathrm{y}^{0}, \mathrm{y}^{1}\right)>0$ under the control constraints (5) is actually due to the positivity of the constant target $\mathrm{y}^{1}$, independently of the initial datum $\mathrm{y}^{0}$.

Theorem 2 and the above remarks are proved in Section 2.5. As discussed previously, this result yields a positive minimal time as well for the control of the Dirichlet heat equation under nonnegativity state constraints.

In Section 2.6 further, we provide lower estimates of the minimal time $\underline{T}\left(\mathrm{y}^{0}, \mathrm{y}^{1}\right)$ when the initial datum $\mathrm{y}^{0}$ is a positive constant.

Before proving Theorem 1, Proposition 1 and Theorem 2, let us recall hereafter the notion of weak solutions with controls that are Radon measures. 


\subsection{Weak solutions with controls that are Radon measures}

We recall here the concept of solution $y$ of the Dirichlet control problem (1) with controls in the (Banach) space of Radon measures $\mathcal{M}(0, T)$ endowed with the norm

$$
\|\mu\|_{\mathcal{M}(0, T)}=\sup \left\{\int_{[0, T]} \varphi(t) \mathrm{d} \mu(t)\left|\varphi \in C^{0}([0, T], \mathbb{R}), \max _{[0, T]}\right| \varphi \mid=1\right\} .
$$

Solutions of the Dirichlet control problem (1) with controls in $\mathcal{M}(0, T)$ are then defined by transposition: given $\mathrm{y}^{0} \in L^{2}(0,1)$, given $u_{0}$ and $u_{1}$ in $\mathcal{M}(0, T)$, we say that $y$ is a weak solution of (1) if

$$
\begin{aligned}
\int_{0}^{T} \int_{0}^{1}\left(-\partial_{t} \varphi(t, x)-\partial_{x}^{2} \varphi(t, x)\right) y(t, x) \mathrm{d} x \mathrm{~d} t-\int_{0}^{1} \mathrm{y}^{0}(x) \varphi(0, x) \mathrm{d} x \\
=\int_{[0, T]} \partial_{x} \varphi(t, 0) \mathrm{d} u_{0}(t)-\int_{[0, T]} \partial_{x} \varphi(t, 1) \mathrm{d} u_{1}(t),
\end{aligned}
$$

for every $\varphi \in C^{2}([0, T] \times[0,1])$ satisfying $\varphi(t, 0)=\varphi(t, 1)=\varphi(T, x)=0$ for all $(t, x) \in[0, T] \times$ $[0,1]$.

Existence and uniqueness of solutions $y \in C^{0}\left([0, T] ; H^{-s}(0,1)\right)$ of $(1)$, for $s>3 / 2$, with controls in $L^{1}(0, T)$, is obtained by classical duality and transposition arguments. This regularity property suffices to give a sense to the trace of the solution at $t=T$ and to the controllability problem when the controls are in $L^{1}(0, T)$. To check this regularity property, it suffices to observe that the solutions of the forced adjoint problem

$$
\begin{aligned}
-\partial_{t} \varphi(t, x) & =\partial_{x}^{2} \varphi(t, x)+f(t, x) & & (t \in(0, T), x \in(0,1)), \\
\varphi(t, 0) & =\varphi(t, 1)=0 & & (t \in(0, T)), \\
\varphi(T, x) & =0 & & (x \in(0,1)),
\end{aligned}
$$

satisfy $\varphi_{x}(0, t), \varphi_{x}(1, t) \in C^{0}([0, T])$ when $f \in L^{1}\left(0, T ; H^{s}(0,1)\right)$ with $s>3 / 2$. This is due to the fact that $\varphi \in C^{0}\left([0, T] ; H^{s}(0,1)\right)$ and that $H^{s}(0,1)$ is continuously embedded in $C^{1}(0,1)$ when $s>3 / 2$.

When the controls are taken to be in $\mathcal{M}(0, T)$, by density arguments, the solution defined by transposition can be shown to be in $L^{\infty}\left(0, T ; H^{-s}(0,1)\right)$ for every $s>3 / 2$. In this case the trace of the solution at $t=0$ and $t=T$ has to be understood in the sense of (6).

The spectral expansion of the solutions of the controlled problem provides an alternative way of representing the solutions given by transposition as above. Indeed, consider the eigenbasis $(\sqrt{2} \sin (n \pi x))_{n \in \mathbb{N}^{*}}$ of the Dirichlet-Laplacian. For every $t \geqslant 0$ and every $n \in \mathbb{N}^{*}$, we set

$$
y_{n}(t)=\int_{0}^{1} y(t, x) \sin (n \pi x) \mathrm{d} x .
$$

Then, by integrating by parts, we easily have

$$
\dot{y}_{n}(t)=n \pi\left(u_{0}(t)-(-1)^{n} u_{1}(t)\right)-n^{2} \pi^{2} y_{n}(t)
$$

with $y_{n}(0)=\int_{0}^{1} \mathrm{y}^{0}(x) \sin (n \pi x) \mathrm{d} x=\mathrm{y}_{n}^{0}$, and thus,

$$
y_{n}(t)=e^{-n^{2} \pi^{2} t} \mathrm{y}_{n}^{0}+n \pi \int_{[0, t]} e^{-n^{2} \pi^{2}(t-s)} \mathrm{d}\left(u_{0}-(-1)^{n} u_{1}\right)(s) .
$$

Then, when the controls $u_{0}$ and $u_{1}$ belong to $\mathcal{M}(0, T)$, at the final time $t=T$ we have

$$
y_{n}(T)=e^{-n^{2} \pi^{2} T} \mathrm{y}_{n}^{0}+n \pi \int_{[0, T]} e^{-n^{2} \pi^{2}(T-s)} \mathrm{d}\left(u_{0}-(-1)^{n} u_{1}\right)(s),
$$

which is well defined in view of the fact that $e^{-n^{2} \pi^{2}(T-s)}$ depends continuously on $s \in(0, T)$. This gives a sense to the trace of the transposition solution at the final time $t=T$. 


\subsection{Proof of Theorem 1}

Let us first mention that this result directly follows from [18, Theorem 4.1, b]. However, we provide a sketch of the proof, showing moreover that the controls can even be chosen in $H^{1}(0, T)$.

First of all, observe that, by subtracting $\mathrm{y}^{1}$, it suffices to prove that there exists a time $T>0$ and controls $u_{0}$ and $u_{1}$ in $H^{1}(0, T)$ satisfying $u_{0}(t)>-\mathrm{y}^{1}$ and $u_{1}(t)>-\mathrm{y}^{1}$ on $[0, T]$, such that the corresponding solution $y$ of $(1)$, with $y(0)=\mathrm{y}^{0}-\mathrm{y}^{1}$, satisfies $y(T)=0$.

According to [8, Theorem 3.3], for any $T>0$ there exist controls $u_{0}, u_{1} \in H^{1}(0, T)$ such that the solution $y$ of (1) for any initial condition in $L^{2}(0,1)$ satisfies $y(T, \cdot)=0$.

Note that, at this point, we use the classical controllability property for the $1 \mathrm{D}$ heat equation without any constraint. Our goal is to show that, if the control time $T>0$ is large enough, then the controls can be taken such that $u_{0}(t)>-\mathrm{y}^{1}$ and $u_{1}(t)>-\mathrm{y}^{1}$ for almost every $t \in[0, T]$.

It is well known (see [14]) that controllability with controls in $H^{1}(0, T)$, without any constraint, is equivalent to an observability inequality, namely to the existence of an observability constant $c(T)>0$ such that

$$
\|z(0, \cdot)\|_{L^{2}(0,1)}^{2} \leqslant c(T)\left(\left\|\partial_{x} z(\cdot, 0)\right\|_{H^{-1}(0, T)}^{2}+\left\|\partial_{x} z(\cdot, 1)\right\|_{H^{-1}(0, T)}^{2}\right),
$$

for any solution of the adjoint system

$$
\begin{aligned}
-\partial_{t} z(t, x) & =\partial_{x}^{2} z(t, x) & & (t>0, x \in(0,1)), \\
z(t, 0) & =z(t, 1)=0 & & (t>0),
\end{aligned}
$$

such that $z(T) \in L^{2}(0,1)$. Actually, the controllability (and equivalently, the observability of the adjoint system) being true on any time interval $(\tau, T)$, we also have

$$
\|z(\tau, \cdot)\|_{L^{2}(0,1)}^{2} \leqslant c(T-\tau)\left(\left\|\partial_{x} z(\cdot, 0)\right\|_{H^{-1}(\tau, T)}^{2}+\left\|\partial_{x} z(\cdot, 1)\right\|_{H^{-1}(\tau, T)}^{2}\right) .
$$

Using a spectral expansion and the Parseval equality, we have the estimate

$$
\|z(0, \cdot)\|_{L^{2}(0,1)}^{2} \leqslant e^{-2 \pi^{2} \tau}\|z(\tau, \cdot)\|_{L^{2}(0,1)}^{2}
$$

for every $0<\tau<T$, and hence

$$
\|z(0, \cdot)\|_{L^{2}(0,1)}^{2} \leqslant e^{-2 \pi^{2} \tau} c(T-\tau)\left(\left\|\partial_{x} z(\cdot, 0)\right\|_{H^{-1}(0, T)}^{2}+\left\|\partial_{x} z(\cdot, 1)\right\|_{H^{-1}(0, T)}^{2}\right) .
$$

By duality, this means that the controls $u_{0}$ and $u_{1}$ can be chosen such that

$$
\left\|u_{i}\right\|_{H^{1}(0, T)}^{2} \leqslant e^{-2 \pi^{2} \tau} c(T-\tau)\left\|\mathrm{y}^{0}-\mathrm{y}^{1}\right\|_{L^{2}(0,1)}^{2} \quad i=0,1,
$$

for any $0<\tau<T$. By continuous embedding of $H^{1}(0, T)$ into $L^{\infty}(0, T)$,

$$
\left\|u_{i}\right\|_{L^{\infty}(0, T)}^{2} \leqslant C\left\|u_{i}\right\|_{H^{1}(0, T)} \leqslant C(T) e^{-2 \pi^{2} \tau} c_{1}(T-\tau)\left\|\mathrm{y}^{0}-\mathrm{y}^{1}\right\|_{L^{2}(0,1)}^{2} \quad i=0,1,
$$

with $C(T)$ the constant of the continuous embedding. Hence, taking $\tau=T / 2$ and observing that $c_{1}(T-\tau)=c_{1}(T / 2)$ is monotonic decreasing with respect to $T$ and observing that $C(T)$ is bounded by a polynomial function, we have, for $T$ large enough,

$$
\left\|u_{0}\right\|_{L^{\infty}(0, T)},\left\|u_{1}\right\|_{L^{\infty}(0, T)}<\mathrm{y}^{1}
$$

and hence $u_{0}(t)>-\mathrm{y}^{1}$ and $u_{1}(t)>-\mathrm{y}^{1}$ on $[0, T]$. This ends the proof of Theorem 1 . 


\subsection{Proof of Proposition 1}

First of all according to Theorem 1, for $T$ large enough there exist controls $u_{0} \in L^{1}(0, T)$ and $u_{1} \in L^{1}(0, T)$, satisfying the constraints (5), such that the corresponding solution $y$ of (1), with $y(0)=\mathrm{y}^{0}$, satisfies $y(T)=\mathrm{y}^{1}$.

As in the proof of Theorem 1, it suffices to prove that there exist controls $\hat{v}_{0}$ and $\hat{v}_{1}$ in $C^{n}([0, T+\tau])$ satisfying $\hat{v}_{0}(t)>-\mathrm{y}^{1}$ and $\hat{v}_{1}(t)>-\mathrm{y}^{1}$ on $[0, T+\tau]$, such that the corresponding solution $\hat{y}$ of $(1)$, with $\hat{y}(0)=\mathrm{y}^{0}-\mathrm{y}^{1}$, satisfies $\hat{y}(T+\tau)=0$. Note first that the controls $v_{0}=u_{0}-\mathrm{y}^{1}$ and $v_{1}=u_{1}-\mathrm{y}^{1}$ satisfy $v_{0}(t)>-\mathrm{y}^{1}$ and $v_{1}(t)>-\mathrm{y}^{1}$ on $[0, T]$, and the corresponding solution $y$ of (1), with $y(0)=\mathrm{y}^{0}-\mathrm{y}^{1}$, satisfies $y(T)=0$.

The idea of the proof is the following. Firstly, we will smoothen the controls $v_{0}$ and $v_{1}$. Then the solution of the heat equation at time $T$, obtained with these smooth controls, will be some $\overline{\mathrm{y}}^{1}$, close to 0 . Secondly, we will steer $\overline{\mathrm{y}}^{1}$ to the target 0 in time $\tau$ with smooth controls. Of course, in order to ensure that the controls steering $\overline{\mathrm{y}}^{1}$ to 0 in time $\tau$ are greater than $-\mathrm{y}^{1}$, the smaller $\tau$ is, the more $\overline{\mathrm{y}}^{1}$ must be close to 0 .

For every $\varepsilon>0$, by a density argument, there exist $\bar{v}_{0}^{\varepsilon}$ and $\bar{v}_{1}^{\varepsilon}$ in $C^{\infty}(0, T)$ such that $\left\|v_{i}-\bar{v}_{i}^{\varepsilon}\right\|_{L^{1}(0, T)}<\varepsilon$, and such that all time derivatives of $\bar{v}_{0}^{\varepsilon}$ and $\bar{v}_{1}^{\varepsilon}$ vanish at time $T$. Since $v_{0} \geqslant-\mathrm{y}^{1}$ and $v_{1} \geqslant-\mathrm{y}^{1}$, we assume moreover that $\bar{v}_{0}^{\varepsilon} \geqslant-\mathrm{y}^{1}$ and $\bar{v}_{1}^{\varepsilon} \geqslant-\mathrm{y}^{1}$.

The well posedness of the heat equation for $L^{1}$ Dirichlet boundary conditions (see Section 2.2) ensures that the solution $\bar{y}$ of (1), with $\bar{y}(0)=\mathrm{y}^{0}-\mathrm{y}^{1}$ and boundary controls $\bar{v}_{0}^{\varepsilon}$ and $\bar{v}_{1}^{\varepsilon}$, satisfies $\|\bar{y}(T)\|_{H^{-2}(0,1)} \leqslant C_{1} \varepsilon$, for some $C_{1}>0$.

Set $\overline{\mathrm{y}}^{1}=\bar{y}(T) \in H^{-2}(0,1)$ and set $\overline{\mathrm{y}}^{0}=y_{0}(\tau / 2) \in L^{2}(0,1)$, where $y_{0}$ is the solution of (1) corresponding to the initial condition $y_{0}(0)=\overline{\mathrm{y}}^{1}$ and to null boundary controls. Then there exists $C_{2}(\tau)>0$ such that $\left\|\overline{\mathrm{y}}^{0}\right\|_{L^{2}(0,1)} \leqslant C_{2}(\tau)\left\|\overline{\mathrm{y}}^{1}\right\|_{H^{-2}(0,1)}$, i.e., $\left\|\overline{\mathrm{y}}^{0}\right\|_{L^{2}(0,1)} \leqslant C_{1} C_{2}(\tau) \varepsilon$.

The aim is now to steer $\overline{\mathrm{y}}^{0}$ to 0 with controls $\tilde{v}_{0}$ and $\tilde{v}_{1}$ in $C^{n}([0, \tau / 2])$ such that

$$
\tilde{v}_{i}(t) \geqslant-\mathrm{y}^{1} \quad(t \in[0, \tau / 2]) \quad \text { and } \quad v_{i}(0)=\cdots=v_{i}^{(n)}(0)=0 \quad(i=0,1) .
$$

This is a consequence of the following lemma.

Lemma 1. Let $\mathrm{y}^{0} \in L^{2}(0,1)$, let $n \in \mathbb{N}^{*}$ and let $\tau>0$ be arbitrary. There exist controls $v_{0}$ and $v_{1}$ in $C^{n}([0, \tau])$ satisfying $v_{i}(0)=\cdots=v_{i}^{(n)}(0)=0$, for $i=1,2$, such that the corresponding solution of (1) (with controls $v_{0}$ and $v_{1}$ ) with initial condition $y(0)=\mathrm{y}^{0}$ satisfies $y(\tau)=0$. Moreover, $v_{0}$ and $v_{1}$ can be chosen such that

$$
\max \left(\left\|v_{0}\right\|_{L^{\infty}(0, \tau)},\left\|v_{1}\right\|_{L^{\infty}(0, \tau)}\right) \leqslant \kappa_{n}(\tau)\left\|\mathrm{y}^{0}\right\|_{L^{2}(0,1)},
$$

for some $\kappa_{n}(\tau)>0$ only depending on $\tau$ and $n$.

Proof of Lemma 1. Pick a function $\rho \in C^{\infty}([0, T])$ such that $\rho(t) \in[0,1], \rho(t)=1$ for $t \in$ $[\tau / 2, \tau]$ and all derivatives of $\rho$ vanish at 0 . We will prove that there exist two functions $u_{0}$ and $u_{1}$ in $H^{n+1}(0, \tau)$ such that the solution $y$ of (1) with initial condition $y(0)=\mathrm{y}^{0}$ and controls $v_{0}=\rho u_{0}$ and $v_{1}=\rho u_{1}$ satisfies $y(\tau)=0$. Consequently, we will have $v_{0}$ and $v_{1}$ in $C^{n}(0, \tau)$ and $v_{i}(0)=\cdots=v_{i}^{(n)}(0)=0$, for $i=0,1$.

In order to prove this fact, we will establish an observability inequality for the adjoint problem, namely, that there exists $c_{n}(\tau)>0$ only depending on $\tau$ and $n$ such that

$$
\|z(0, \cdot)\|_{L^{2}(0,1)} \leqslant c_{n}(\tau)\left(\left\|\rho^{2} \partial_{x} z(\cdot, 0)\right\|_{H^{-(n+1)}(0, \tau)}+\left\|\rho^{2} \partial_{x} z(\cdot, 1)\right\|_{H^{-(n+1)}(0, \tau)}\right),
$$

where $z$ is solution of the adjoint problem (7).

But, according to [8, Theorem 3.3], for any $T>0$ there exist controls $u_{0}, u_{1} \in H^{n+1}(0, T)$ such that the solution $y$ of (1) for any initial condition in $L^{2}(0,1)$ satisfies $y(T)=0$. That is to say that there exists a constant $c_{n}(\tau)$ such that,

$$
\|z(\tau / 2, \cdot)\|_{L^{2}(0,1)} \leqslant c_{n}(\tau)\left(\left\|\partial_{x} z(\cdot, 0)\right\|_{H^{-(n+1)}(\tau / 2, \tau)}+\left\|\partial_{x} z(\cdot, 1)\right\|_{H^{-(n+1)}(\tau / 2, \tau)}\right) .
$$


But we have,

$$
\left\|\partial_{x} z(\cdot, x)\right\|_{H^{-(n+1)}(\tau / 2, \tau)} \leqslant\left\|\rho^{2} \partial_{x} z(\cdot, x)\right\|_{H^{-(n+1)}(0, \tau)} \quad(x \in\{0,1\})
$$

and hence,

$$
\|z(\tau / 2, \cdot)\|_{L^{2}(0,1)} \leqslant c_{n}(\tau)\left(\left\|\rho^{2} \partial_{x} z(\cdot, 0)\right\|_{H^{-(n+1)}(0, \tau)}+\left\|\rho^{2} \partial_{x} z(\cdot, 1)\right\|_{H^{-(n+1)}(0, \tau)}\right) .
$$

Due to the dissipativity properties of the heat equation, we have

$$
\|z(0, \cdot)\|_{L^{2}(0,1)} \leqslant e^{-\pi^{2} \tau / 2}\|z(\tau / 2, \cdot)\|_{L^{2}(0,1)}
$$

and hence,

$$
\|z(0, \cdot)\|_{L^{2}(0,1)} \leqslant e^{-\pi^{2} \tau / 2} c_{n}(\tau)\left(\left\|\rho^{2} \partial_{x} z(\cdot, 0)\right\|_{H^{-(n+1)}(0, \tau)}+\left\|\rho^{2} \partial_{x} z(\cdot, 1)\right\|_{H^{-(n+1)}(0, \tau)}\right) .
$$

The latter inequality ensures that there exist two functions $u_{0}$ and $u_{1}$ in $H^{n+1}(0, \tau)$ such that the solution $y$ of (1) with initial condition $y(0)=\mathrm{y}^{0}$ and controls $v_{0}=\rho u_{0}$ and $v_{1}=\rho u_{1}$ satisfies $y(\tau)=0$. By duality, this also means that $u_{0}$ and $u_{1}$ can be chosen such that

$$
\left\|u_{i}\right\|_{H^{n+1}(0, \tau)} \leqslant e^{-\pi^{2} \tau / 2} c_{n}(\tau)\left\|\mathrm{y}^{0}\right\|_{L^{2}(0,1)} \quad(i=0,1) .
$$

Since $H^{n+1}(0, \tau)$ is continuously embedded in $L^{\infty}(0, \tau)$, with embedding constant $C_{n}(\tau)$, for any $n \geqslant 0$, we infer that

$$
\left\|u_{i}\right\|_{L^{\infty}(0, \tau)} \leqslant C_{n}(\tau)\left\|u_{i}\right\|_{H^{n+1}(0, \tau)} \leqslant e^{-\pi^{2} \tau / 2} C_{n}(\tau) c_{n}(\tau)\left\|\mathrm{y}^{0}\right\|_{L^{2}(0,1)} \quad(i=0,1) .
$$

Since $\rho(t) \in[0,1]$, we get $\left\|v_{i}\right\|_{L^{\infty}(0, \tau)} \leqslant\left\|u_{i}\right\|_{L^{\infty}(0, \tau)}$.

Lemma 1 is proved.

We are now in a position to conclude the proof of Proposition 1. According to Lemma 1, given any $\tau>0$, and $\overline{\mathrm{y}}^{0} \in L^{2}(0,1)$, there exist controls $\tilde{v}_{0}$ and $\tilde{v}_{0}$ in $C^{n}([0, \tau / 2])$ satisfying $\tilde{v}_{0}(0)=\tilde{v}_{1}(0)=\cdots=\tilde{v}_{0}^{(n)}(0)=\tilde{v}_{1}^{(n)}(0)=0$, such that the solution $\tilde{y}$ of the heat process $(1)$ with initial condition $\tilde{y}(0)=\overline{\mathrm{y}}^{0}$ and Dirichlet boundary controls $\tilde{v}_{0}$ and $\tilde{v}_{1}$ satisfies $\tilde{y}(\tau / 2)=0$. Moreover, we have

$$
\inf _{(0, \tau / 2)} \tilde{v}_{i} \geqslant-\kappa_{n}(\tau / 2)\left\|\overline{\mathrm{y}}^{0}\right\|_{L^{2}(0,1)} \quad(i=0,1) .
$$

But, at the beginning of this proof, it has been shown that $\left\|\overline{\mathrm{y}}^{0}\right\|_{L^{2}(0,1)} \leqslant C_{1} C_{2}(\tau) \varepsilon$, where $\varepsilon>0$ can be chosen arbitrary small. Consequently, for $\varepsilon<\varepsilon(n, \tau)=\mathrm{y}^{1} / C_{1} C_{2}(\tau) \kappa_{n}(\tau)$, we have $\tilde{v}_{i}(t) \geqslant-\mathrm{y}^{1}$ for $i=0,1$ and for almost $t \in(0, \tau / 2)$.

All in all, for this small enough $\varepsilon>0$, we set

$$
\hat{v}_{i}(t)=\left\{\begin{array}{ll}
\bar{v}_{i}^{\varepsilon}(t) & \text { if } t \in(0, T) \\
0 & \text { if } t \in(T, T+\tau / 2) \\
\tilde{v}_{i}(t-T-\tau / 2) & \text { if } t \in(T+\tau / 2, T+\tau)
\end{array} \quad(i=0,1)\right.
$$

Consequently, we have $\hat{v}_{0}$ and $\hat{v}_{1}$ in $C^{n}([0, T+\tau]), \hat{v}_{0}(t) \geqslant-\mathrm{y}^{1}$ and $\hat{v}_{1}(t) \geqslant-\mathrm{y}^{1}$ and the solution $\hat{y}$ of (1) with Dirichlet controls $\hat{v}_{0}$ and $\hat{v}_{1}$ and initial condition $\hat{y}(0)=\mathrm{y}^{0}-\mathrm{y}^{1}$ satisfies $\hat{y}(T+\tau)=0$. Proposition 1 is proved. 


\subsection{Proof of Theorem 2}

Positivity of $\underline{T}\left(\mathrm{y}^{0}, \mathrm{y}^{1}\right)$. In view of the spectral decomposition given in Section 2.2, the fact that controls $\mathrm{z} u_{0}$ and $u_{1}$ steer the solution $y$ from $\mathrm{y}^{0}$ to $\mathrm{y}^{1}$ in time $T$, is equivalent to

$$
y_{n}(T)=\int_{0}^{1} \mathrm{y}^{1} \sin (n \pi x) \mathrm{d} x=\frac{1-(-1)^{n}}{n \pi} \mathrm{y}^{1}, \forall n \geq 1,
$$

and thus,

$$
\frac{1-(-1)^{n}}{n \pi} \mathrm{y}^{1}-e^{-n^{2} \pi^{2} T} \mathrm{y}_{n}^{0}=n \pi \int_{[0, T]} e^{-n^{2} \pi^{2}(T-t)} \mathrm{d}\left(u_{0}-(-1)^{n} u_{1}\right)(t), \quad \forall n \geq 1 .
$$

In particular, for $n=2 p$,

$$
\int_{[0, T]} e^{(2 p)^{2} \pi^{2} t} \mathrm{~d}\left(u_{0}-u_{1}\right)(t)=-\frac{\mathrm{y}_{2 p}^{0}}{2 p \pi},
$$

and for $n=2 p+1$,

$$
\frac{2 \mathrm{y}^{1}}{(2 p+1) \pi}-e^{-(2 p+1)^{2} \pi^{2} T} \mathrm{y}_{2 p+1}^{0}=(2 p+1) \pi \int_{[0, T]} e^{-(2 p+1)^{2} \pi^{2}(T-t)} \mathrm{d}\left(u_{0}+u_{1}\right)(t) .
$$

But, for every $t \in[0, T]$, we have $e^{-(2 p+1)^{2} \pi^{2} T} \leqslant e^{-(2 p+1)^{2} \pi^{2}(T-t)} \leqslant 1$. Consequently, assuming that $u_{0}$ and $u_{1}$ are nonnegative controls, we obtain, for every $p \in \mathbb{N}$,

$$
\begin{aligned}
e^{-(2 p+1)^{2} \pi^{2} T} \int_{[0, T]} \mathrm{d}\left(u_{0}+u_{1}\right)(t) \leqslant \int_{[0, T]} e^{-(2 p+1)^{2} \pi^{2}(T-t)} \mathrm{d}\left(u_{0}+u_{1}\right)(t) & \\
& \leqslant \int_{[0, T]} \mathrm{d}\left(u_{0}+u_{1}\right)(t),
\end{aligned}
$$

that is,

$$
\begin{aligned}
& (2 p+1) \pi e^{-(2 p+1)^{2} \pi^{2} T} \int_{[0, T]} \mathrm{d}\left(u_{0}+u_{1}\right)(t) \\
& \qquad \frac{2 \mathrm{y}^{1}}{(2 p+1) \pi}-e^{-(2 p+1)^{2} \pi^{2} T} \mathrm{y}_{2 p+1}^{0} \leqslant(2 p+1) \pi \int_{[0, T]} \mathrm{d}\left(u_{0}+u_{1}\right)(t)
\end{aligned}
$$

and hence,

$$
\begin{aligned}
\frac{2 \mathrm{y}^{1}}{(2 p+1)^{2} \pi^{2}}-e^{-(2 p+1)^{2} \pi^{2} T} \frac{\mathrm{y}_{2 p+1}^{0}}{(2 p+1) \pi} \leqslant \int_{[0, T]} \mathrm{d}( & \left.u_{0}+u_{1}\right)(t) \\
& \leqslant e^{(2 p+1)^{2} \pi^{2} T} \frac{2 \mathrm{y}^{1}}{(2 p+1)^{2} \pi^{2}}-\frac{\mathrm{y}_{2 p+1}^{0}}{(2 p+1) \pi} .
\end{aligned}
$$

Now, assume by contradiction that for every $T>0$ there exist nonnegative controls $u_{0}^{T}$ and $u_{1}^{T}$ steering $\mathrm{y}^{0}$ to $\mathrm{y}^{1}$ in time $T$. Then, (9) ensures that $\lim _{T \rightarrow 0} \int_{[0, T]} \mathrm{d}\left(u_{0}^{T}+u_{1}^{T}\right)(t)$ exists and we have

$$
\lim _{T \rightarrow 0} \int_{[0, T]} \mathrm{d}\left(u_{0}^{T}+u_{1}^{T}\right)(t)=\frac{2 \mathrm{y}^{1}}{(2 p+1)^{2} \pi^{2}}-\frac{\mathrm{y}_{2 p+1}^{0}}{(2 p+1) \pi} \quad(p \in \mathbb{N}) .
$$

Then, by uniqueness of the limit, necessarily, there exists $\gamma \in \mathbb{R}$ such that $\gamma=\frac{2 \mathrm{y}^{1}}{(2 p+1)^{2} \pi^{2}}-$ $\frac{\mathrm{y}_{2 p+1}^{0}}{(2 p+1) \pi}$ for every $p \in \mathbb{N}$, and therefore $\mathrm{y}_{2 p+1}^{0}=\frac{2 \mathrm{y}^{1}}{(2 p+1) \pi}-(2 p+1) \pi \gamma$ for every $p \in \mathbb{N}$. Since $\mathrm{y}^{0} \in L^{2}(0,1)$ and thus $\sum_{n=0}^{\infty}\left|\mathrm{y}_{n}^{0}\right|^{2}<\infty$, we must have $\gamma=0$, and hence $\mathrm{y}_{2 p+1}^{0}=\frac{2 \mathrm{y}^{1}}{(2 p+1) \pi}$ for 
every $p \in \mathbb{N}$, and $\int_{[0, T]} \mathrm{d}\left(u_{0}^{T}+u_{1}^{T}\right) \rightarrow 0$ as $T \rightarrow 0$. Since $u_{0}^{T}$ and $u_{1}^{T}$ are nonnegative, we also conclude that $\int_{[0, T]} \mathrm{d} u_{0}^{T} \rightarrow 0$ and $\int_{[0, T]} \mathrm{d} u_{1}^{T} \rightarrow 0$ as $T \rightarrow 0$. Letting $T$ tend to 0 in (8a), we obtain $\mathrm{y}_{2 p}^{0}=0$ for every $p \in \mathbb{N}^{*}$.

All in all, since the family $(\sqrt{2} \sin (n \pi \cdot))_{n \in \mathbb{N}^{*}}$ is an orthonormal basis of $L^{2}(0,1)$, taking into account that $\mathrm{y}_{2 p+1}^{0}=\frac{2 \mathrm{y}^{1}}{(2 p+1) \pi}$ and $\mathrm{y}_{2 p}^{0}=0$ for every $p$, we conclude that $\mathrm{y}^{0}$ can be steered to $\mathrm{y}^{1}$ in arbitrarily small time with nonnegative controls if and only if $\mathrm{y}^{0}=\mathrm{y}^{1}$. This proves the first part of the theorem.

Constrained controllability at the minimal time $\underline{T}=\underline{T}\left(\mathrm{y}^{0}, \mathrm{y}^{1}\right)$. Let us prove the existence of measure-valued nonnegative controls realizing the controllability exactly in time $T$.

In view of the definition of the minimal control time as the infimum of positive times $T$ for which there exist controls $u_{0}$ and $u_{1}$ in $L^{1}(0, T)$, for every $n \in \mathbb{N}$, there exist controls $u_{0}^{n}$ and $u_{1}^{n}$ in $L^{1}(0, \underline{T}+1 / n)$ satisfying the constraints (5), such that the corresponding solution $y$ of $(1)$, with $y(\overline{0})=\mathrm{y}^{0}$, satisfies $y(\underline{T}+1 / n)=\mathrm{y}^{1}$. We extend the controls $u_{0}^{n}$ and $u_{1}^{n}$ by 0 on $(T+1 / n, T+1)$. According to (9), we have, for every $n \in \mathbb{N}$,

$$
\begin{aligned}
\left\|u_{0}^{n}\right\|_{L^{1}(0, \underline{T}+1)}+\left\|u_{1}^{n}\right\|_{L^{1}(0, \underline{T}+1)} & =\int_{0}^{\underline{T}+1 / n}\left(u_{0}(t)+u_{1}(t)\right) \mathrm{d} t \\
& \leqslant \inf _{p \in \mathbb{N}}\left(e^{(2 p+1)^{2} \pi^{2} \underline{T}} \frac{2 \mathrm{y}^{1}}{(2 p+1)^{2} \pi^{2}}-\frac{\mathrm{y}_{2 p+1}^{0}}{(2 p+1) \pi}\right) \leqslant \frac{2 e^{\pi^{2} \underline{T}} \mathrm{y}^{1}}{\pi^{2}}-\frac{\mathrm{y}_{1}^{0}}{\pi}<+\infty .
\end{aligned}
$$

Then, the sequences $\left(u_{0}^{n}\right)_{n}$ and $\left(u_{1}^{n}\right)_{n}$ are bounded in $L^{1}(0, \underline{T}+1)$ and therefore, by weak compactness of $\mathcal{M}(0, T)$, up to a subsequence they converge in the weak sense of Radon measures to some controls $\underline{u}_{i}$ in $\mathcal{M}(0, T)$.

Thanks to the well-posedness results recalled at the beginning of the section, ensuring that the corresponding solutions are bounded in $C^{0}\left([0, \underline{T}], H^{-s}(0,1)\right)$ for any $s>3 / 2$, we can pass to limit (extracting subsequences) so that the controls converge weakly in the sense of measures in $\mathcal{M}(0, T)$ and the corresponding solutions in the weak-* topology of $L^{\infty}\left([0, T], H^{-s}(0,1)\right)$ for any $s>3 / 2$. Clearly, the limit controls satisfy the nonnegativity constraint and the limit solution solves the limit non-homogeneous Dirichlet problem (1) in the sense of transposition (6). The limit solution reaches the target $\mathrm{y}^{1}$ in time $\underline{T}$.

Proof of the statement in Remark 2. Assuming that $\mathrm{y}^{0}(x)=\mathrm{y}^{0}(1-x)$ for almost every $x \in[0,1]$, we claim that we can take $u_{0}=u_{1}$. Indeed, it is easy to see that, given any pair $\left(u_{0}, u_{1}\right)$ of controls realizing the controllability at some arbitrary time $T \geqslant \underline{T}$, the pair $\left(\left(u_{0}+u_{1}\right) / 2,\left(u_{0}+u_{1}\right) / 2\right)$ satisfies the same conclusion. The statement follows.

\subsection{Lower estimates of the minimal time for a constant initial datum}

In this section, we assume moreover that $\mathrm{y}^{0}>0$ is a positive constant. The arguments of the proof given in the previous section allow to derive lower estimates of the waiting time $\underline{T}\left(\mathrm{y}^{0}, \mathrm{y}^{1}\right)$.

Lemma 2. Let $\mathrm{y}^{0}$ and $\mathrm{y}^{1}$ be positive real numbers such that $\mathrm{y}^{0} \neq \mathrm{y}^{1}$. We set $\underline{T}=\underline{T}\left(\mathrm{y}^{0}, \mathrm{y}^{1}\right)$.

1. If $\mathrm{y}^{1}<\mathrm{y}^{0}$ then

$$
\underline{T}>\frac{1}{\pi^{2}} \ln \frac{\mathrm{y}^{0}}{\mathrm{y}^{1}} \quad \text { and } \quad \sup _{p \in \mathbb{N}^{*}} \frac{1}{(2 p+1)^{2}}\left(\frac{\mathrm{y}^{1}}{\mathrm{y}^{0}}-e^{-(2 p+1)^{2} \pi^{2} \underline{T}}\right) \leqslant \frac{\mathrm{y}^{1}}{\mathrm{y}^{0}} e^{\pi^{2} \underline{T}}-1 .
$$


2. If $\mathrm{y}^{1}>\mathrm{y}^{0}$ then

$$
\frac{\mathrm{y}^{1}}{\mathrm{y}^{0}}-e^{-\pi^{2} \underline{T}} \leqslant \inf _{p \in \mathbb{N}^{*}} \frac{1}{(2 p+1)^{2}}\left(\frac{\mathrm{y}^{1}}{\mathrm{y}^{0}} e^{(2 p+1)^{2} \pi^{2} \underline{T}}-1\right) .
$$

Proof. By Remark 2, we can take $u_{0}=u_{1}$. Using the notations of the proof of Theorem 2, since $\mathrm{y}^{0}$ is constant, we have $\mathrm{y}_{n}^{0}=\frac{1-(-1)^{n}}{n \pi} \mathrm{y}^{0}$, for every $n \in \mathbb{N}^{*}$, and then (9) gives

$$
\frac{2\left(\mathrm{y}^{1}-e^{-(2 p+1)^{2} \pi^{2} T} \mathrm{y}^{0}\right)}{(2 p+1)^{2} \pi^{2}} \leqslant 2 \int_{0}^{T} u_{0}(t) \mathrm{d} t \leqslant \frac{2\left(e^{(2 p+1)^{2} \pi^{2} T} \mathrm{y}^{1}-\mathrm{y}^{0}\right)}{(2 p+1)^{2} \pi^{2}},
$$

for every $p \in \mathbb{N}$, which yields

$$
\sup _{p \in \mathbb{N}} \frac{\mathrm{y}^{1}-e^{-(2 p+1)^{2} \pi^{2} T} \mathrm{y}^{0}}{(2 p+1)^{2} \pi^{2}} \leqslant \inf _{p \in \mathbb{N}} \frac{e^{(2 p+1)^{2} \pi^{2} T} \mathrm{y}^{1}-\mathrm{y}^{0}}{(2 p+1)^{2} \pi^{2}} \quad \text { and } \quad \inf _{p \in \mathbb{N}} \frac{e^{(2 p+1)^{2} \pi^{2} T} \mathrm{y}^{1}-\mathrm{y}^{0}}{(2 p+1)^{2} \pi^{2}} \geqslant 0 .
$$

The lemma then follows by simple computations.

Providing more explicit lower estimates is a bit technical. Let us do it however. For all $\delta>1, \mu>1$ and $Z \in[0,+\infty)$, we define

$$
f_{\delta, \mu}(Z)=\frac{\delta}{\mu} Z^{\mu+1}-\left(\delta+\frac{1}{\mu}\right) Z+1 .
$$

Noting that $f_{\delta, \mu}^{\prime}(Z)=\frac{\mu+1}{\mu} \delta Z^{\mu}-\left(\delta+\frac{1}{\mu}\right)$ and that $f_{\delta, \mu}^{\prime \prime}(Z)=\delta(\mu+1) Z^{\mu-1}>0$ for every $Z>0$, it follows that $f_{\delta, \mu}$ is a strictly convex function on $[0,+\infty)$. Since $f_{\delta, \mu}(0)=1>0, f_{\delta, \mu}\left(\frac{1}{\delta}\right)=$ $\frac{1}{\mu}\left(\frac{1}{\delta^{\mu}}-\frac{1}{\delta}\right)<0, f_{\delta, \mu}(1)=\frac{1-\mu}{\mu}(\delta-1)<0$ and $f_{\delta, \mu}(Z) \rightarrow+\infty$ as $Z \rightarrow+\infty$, we infer that there exist $Z_{1, \delta, \mu} \in\left(0, \frac{1}{\delta}\right)$ and $Z_{2, \delta, \mu} \in(1,+\infty)$ such that $f_{\delta, \mu}\left(Z_{1, \delta, \mu}\right)=f_{\delta, \mu}\left(Z_{2, \delta, \mu}\right)=0$, and by convexity, $Z_{1, \delta, \mu}$ and $Z_{2, \delta, \mu}$ are the only roots of $f_{\delta, \mu}$.

We set $\mu_{p}=(2 p+1)^{2} \geqslant 1$, for $p \in \mathbb{N}$. The graphs of $f_{\delta, \mu_{p}}$ for $p \in\{1, \ldots, 5\}$ and $\delta=5$ are drawn on Figure 1. Numerically, we obtain $Z_{1, \delta, \mu_{1}}=0.195652$ and $Z_{2, \delta, \mu_{1}}=1.255783$.

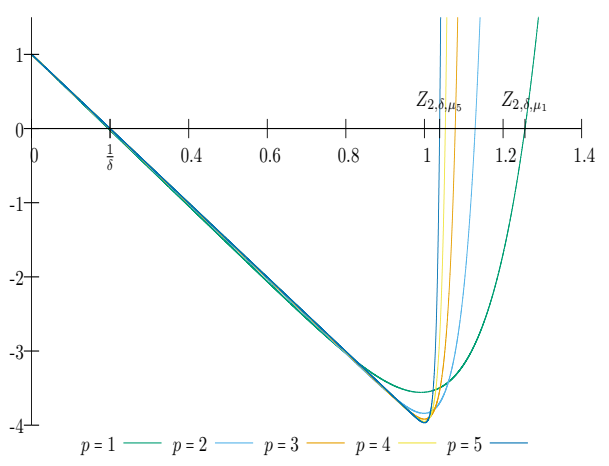

(a) Global view.

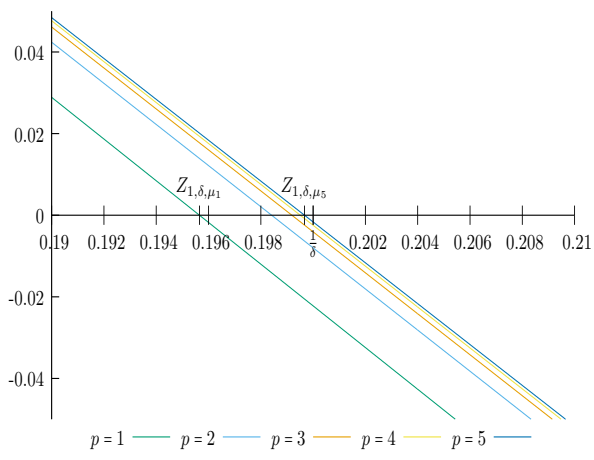

(b) Zoom around $1 / \delta$.

Figure 1: Graphs of $f_{\delta, \mu_{p}}$ for $p \in\{1, \ldots, 5\}$ and $\delta=5$.

Case $\mathrm{y}^{1}<\mathrm{y}^{0}$. Setting $\eta=\frac{\mathrm{y}^{0}}{\mathrm{y}^{1}}>1$ and $Z=\exp \left(-\pi^{2} \underline{T}\right) \in(0,1)$, we have $0<Z<\frac{1}{\eta}$ and $\sup _{p \in \mathbb{N}^{*}} \frac{1}{\mu_{p}}\left(\frac{1}{\eta}-Z^{\mu_{p}}\right) \leqslant \frac{1}{\eta Z}-1$, which gives $\sup _{p \in \mathbb{N}^{*}} f_{\eta, \mu_{p}}(Z) \geqslant 0$ (by multiplying by $-\eta Z$ ). Using the properties of $f_{\delta, \mu}$, this leads to $0<Z \leqslant Z^{*}=\inf _{p \in \mathbb{N}^{*}} Z_{1, \eta, \mu_{p}}$.

We claim that $Z^{*}>0$. Indeed, for $Z \in[0,1)$, we have $f_{\delta, \mu}(Z) \geqslant 1-\left(\delta+\frac{1}{\mu}\right) Z$. By definition, $f_{\delta, \mu}\left(Z_{1, \delta, \mu}\right)=0$ and, consequently, $1-\left(\delta+\frac{1}{\mu}\right) Z_{1, \delta, \mu} \leqslant 0$. Since $Z \mapsto 1-\left(\delta+\frac{1}{\mu}\right) Z$ is decreasing, we conclude that $Z_{1, \delta, \mu} \geqslant \frac{1}{\delta+\frac{1}{\mu}}$. Since $\lim _{\mu \rightarrow+\infty}\left(\delta+\frac{1}{\mu}\right)^{-1}=\delta^{-1}$, the claim follows. 
Since $Z=\exp \left(-\pi^{2} \underline{T}\right)$, we have thus obtained the lower bound

$$
\underline{T} \geqslant \frac{\left|\ln \left(Z^{*}\right)\right|}{\pi^{2}}
$$

Case $\mathrm{y}^{1}>\mathrm{y}^{0}$. Similarly, setting $\delta=\frac{\mathrm{y}^{1}}{\mathrm{y}^{0}}>1$ and $Z=\exp \left(\pi^{2} \underline{T}\right)>1$, we have $\delta-\frac{1}{Z} \leqslant$ $\inf _{p \in \mathbb{N}^{*}} \frac{1}{\mu_{p}}\left(\delta Z^{\mu_{p}}-1\right)$, that is, $0 \leqslant \inf _{p \in \mathbb{N}^{*}} f_{\delta, \mu_{p}}(Z)$, and hence $Z$ must be such that $Z \geqslant Z_{*}=$ $\sup _{p \in \mathbb{N}^{*}} Z_{2, \delta, \mu_{p}}$. We prove similarly that $Z_{\star}>0$, and we obtain for $\underline{T}$ the same lower bound as above, replacing $Z^{*}$ with $Z_{*}$.

Example 1. If $\mathrm{y}^{0}=5$ and $\mathrm{y}^{1}=1$, we have $\eta=5$ and we observe (see Figure 1(b)) that $Z^{*}=\inf _{p \in \mathbb{N}^{*}} Z_{1, \eta, \mu_{p}}=Z_{1, \eta, \mu_{1}}$. Therefore, $\underline{T}\left(\mathrm{y}^{0}, \mathrm{y}^{1}\right) \geqslant \frac{\mid \ln Z_{1, \eta, \mu_{1} \mid}}{\pi^{2}} \simeq 0.165297$.

If $\mathrm{y}^{0}=1$ and $\mathrm{y}^{1}=5$, we have $\delta=5$ and we observe (see Figure $1(a)$ ) that $\sup _{p \in \mathbb{N}^{*}} Z_{2, \eta, \mu_{p}}=$ $Z_{2, \eta, \mu_{1}}$. Therefore, $\underline{T}\left(\mathrm{y}^{0}, \mathrm{y}^{1}\right) \geqslant \frac{\ln Z_{2, \eta, \mu_{1}}}{\pi^{2}} \simeq 0.023076$.

\section{Minimal time for Dirichlet controlled multi-D heat equations under nonnegativity constraints}

\subsection{Heat equation in a ball with nonnegative Dirichlet controls}

Let $D=B(0,1)$ be the unit ball of $\mathbb{R}^{d}$ and consider the Dirichlet control problem

$$
\begin{aligned}
\partial_{t} y(t, x) & =\Delta y(t, x) & & (t>0, x \in D), \\
y(t, x) & =u(t, x) & & (t>0, x \in \partial D),
\end{aligned}
$$

with the initial condition given in $L^{2}(D)$,

$$
y(0, x)=\mathrm{y}^{0}(x) \quad(x \in D) .
$$

The aim is to steer $y$ solution of (11) to a constant target $\mathrm{y}^{1}>0$ with nonnegative controls $u \in L^{2}\left(0, T ; L^{2}(\partial D)\right)$, i.e., under the constraint

$$
u(t, x) \geqslant 0 \quad(t \geqslant 0, x \in \partial D \quad \text { a.e. }) .
$$

Remark 4. Under the assumption $y^{0} \geqslant 0$, the control problem (11) with the nonnegativity control constraint (12) is equivalent to the control problem (11) with the nonnegativity state constraint $y(t, x) \geqslant 0$.

Indeed, due to the (already employed) comparison principle, if the control $u$ is nonnegative and $\mathrm{y}^{0}$ is nonnegative, then the solution of (11) is nonnegative as well. Conversely, if the solution $y$ of (11) is nonnegative, then its trace on $\partial D$ is nonnegative as well.

Theorem 3. Let $\mathrm{y}^{0} \in L^{2}(D)$ and let $\mathrm{y}^{1} \in L^{2}(D)$ be a positive steady-state, i.e., satisfying $\Delta \overline{\mathrm{y}}^{1}=0$ in $D$ and $\overline{\mathrm{y}}=\bar{u}$ on $\partial D$ for some $\bar{u} \in L^{2}(\partial D)$. We assume that $\mathrm{y}^{0} \neq \mathrm{y}^{1}$ and that there exists $\varepsilon>0$ such that $\mathrm{y}^{1}(x) \geqslant \varepsilon$ for every $x \in D$. Then, we have the following results:

- There exist $T>0$ and a nonnegative control $u \in L^{2}\left(0, T, L^{2}(\partial D)\right)$ such that the corresponding solution $y$ of $(11)$, with $y(0)=\mathrm{y}^{0}$, satisfies $y(T)=\mathrm{y}^{1}$.

- Defining the minimal time by

$$
\underline{T}\left(\mathrm{y}^{0}, \mathrm{y}^{1}\right)=\inf \left\{T>0, \exists u \in L^{1}((0, T) \times \partial D) \text { s.t. } u \geqslant 0 \text { and } y(T)=\mathrm{y}^{1}\right\},
$$

we have $\underline{T}\left(\mathrm{y}^{0}, \mathrm{y}^{1}\right)>0$.

- Given any $T>\underline{T}\left(\mathrm{y}^{0}, \mathrm{y}^{1}\right)$ : 
- For every $\tau>0$, there exists a nonnegative control $u \in L^{2}\left(0, T, L^{2}(\partial D)\right)$ satisfying $u \in C^{\infty}((0, T-\tau) \times \partial D)$, such that the corresponding solution $y$ of $(11)$, with $y(0)=$ $\mathrm{y}^{0}$, satisfies $y(T)=\mathrm{y}^{1}$.

- If $\mathrm{y}^{1} \in C^{\infty}(\bar{\Omega})$, then there exists a nonnegative control $u \in C^{\infty}((0, T) \times \partial D)$ such that the corresponding solution $y$ of $(11)$, with $y(0)=\mathrm{y}^{0}$, satisfies $y(T)=\mathrm{y}^{1}$.

- For $T=\underline{T}\left(\mathrm{y}^{0}, \mathrm{y}^{1}\right)$, there exist a nonnegative control $\underline{u} \in \mathcal{M}((0, T) \times \partial D)$ (the space of Radon measures) such that the corresponding solution $y$ of $(11)$, with $y(0)=\mathrm{y}^{0}$, satisfies $y(T)=\mathrm{y}^{1}$.

As in the 1D case, the minimal control time could be defined in other functional control spaces, not necessarily in $L^{1}$. But the regularizing properties of the heat equation ensure that, in fact, the resulting minimal time is the same even if the controls under consideration are restricted to be smooth.

Proof of Theorem 3. The existence of a large enough time $T$ for which there exist a nonnegative control $u$ steering the solution of (11) from $\mathrm{y}^{0}$ to $\mathrm{y}^{1}$ can be easily proved by adapting the proof of Theorem 1 or can be directly obtained from [18, Theorem 4.1 (b)].

Proving that for every $T>\underline{T}\left(\mathrm{y}^{0}, \mathrm{y}^{1}\right)$ there exist a nonnegative and smooth control steering the solution of (11) from $\mathrm{y}^{0}$ to $\mathrm{y}^{1}$ is similar to the proof of Proposition 1.

Now, let us prove that $\underline{T}\left(\mathrm{y}^{0}, \mathrm{y}^{1}\right)>0$. We proceed as in the proof of Theorem 2, but we now consider the (nondecreasing sequence of positive) eigenvalues $\left(\lambda_{n}\right)_{n \in \mathbb{N}^{*}}$ and eigenfunctions $\left(p_{n}\right)_{n \in \mathbb{N}^{*}}$ of the Sturm-Liouville problem, defined by

$$
\begin{array}{ll}
\frac{\mathrm{d}^{2} p_{n}}{\mathrm{~d} r^{2}}(r)+\frac{d-1}{r} \frac{\mathrm{d} p_{n}}{\mathrm{~d} r}(r)=-\lambda_{n} p_{n}(r) & (r \in(0,1)), \\
p_{n}(0)=1, \quad p_{n}(1)=\frac{\mathrm{d} p_{n}}{\mathrm{~d} r}(0)=0, &
\end{array}
$$

Defining $\alpha_{n}=\frac{\mathrm{d} p_{n}}{\mathrm{~d} r}(1)$ and $\varphi_{n}(x)=p_{n}(\|x\|)$ for $x \in D$, we have

$$
\begin{array}{ll}
\Delta \varphi_{n}(x)=-\lambda_{n} \varphi_{n}(x) & (x \in D), \\
\varphi_{n}(x)=0, \quad \nabla \varphi_{n}(x) \cdot \mathrm{n}(x)=\alpha_{n} & (x \in \partial D) .
\end{array}
$$

Let $T>0$ and $u^{T} \in L^{1}((0, T) \times \partial D)$ be a nonnegative control such that the solution $y$ of (11) with initial condition $\mathrm{y}^{0}$ satisfies $y(T)=\mathrm{y}^{1}$. For every $n \in \mathbb{N}^{*}$, we set $y_{n}(t)=$ $\int_{D} y(t, x) \varphi_{n}(x) \mathrm{d} x$. Then, we have

$$
\begin{aligned}
\dot{y}_{n}(t) & =\int_{D} \Delta y(t, x) \varphi_{n}(x) \mathrm{d} x=\int_{\partial D} \nabla y(t, x) \cdot \mathrm{n}(x) \varphi_{n}(x) \mathrm{d} \Gamma_{x}-\int_{D} \nabla y(t, x) \cdot \nabla \varphi_{n}(x) \mathrm{d} x \\
& =-\int_{\partial D} y(t, x) \nabla \varphi_{n}(x) \cdot \mathrm{n}(x) \mathrm{d} \Gamma_{x}+\int_{D} y(t, x) \Delta \varphi_{n}(x) \mathrm{d} x \\
& =-\alpha_{n} \int_{\partial D} u^{T}(t, x) \mathrm{d} \Gamma_{x}-\lambda_{n} \int_{D} y(t, x) \varphi_{n}(x) \mathrm{d} x=-\lambda_{n} y_{n}(t)-\alpha_{n} \int_{\partial D} u^{T}(t, x) \mathrm{d} \Gamma_{x}
\end{aligned}
$$

and hence,

$$
y_{n}(T)=e^{-\lambda_{n} T} y_{n}(0)-\alpha_{n} \int_{0}^{T} e^{-\lambda_{n}(T-t)} \int_{\partial D} u^{T}(t, x) \mathrm{d} \Gamma_{x} \mathrm{~d} t .
$$

Setting $\mathrm{y}_{n}^{i}=\int_{D} \mathrm{y}^{i}(x) \varphi_{n}(x) \mathrm{d} x$ for $i=0,1$, we obtain ( since $u^{T}$ is a control such that $\left.y(T)=\mathrm{y}^{1}\right)$,

$$
\mathrm{y}_{n}^{1}-e^{-\lambda_{n} T} \mathrm{y}_{n}^{0}=-\alpha_{n} \int_{0}^{T} e^{-\lambda_{n}(T-t)} \int_{\partial D} u^{T}(t, x) \mathrm{d} \Gamma_{x} \mathrm{~d} t
$$

Since $u^{T} \geqslant 0$ and $\lambda_{n}>0$, we obtain

$$
e^{-\lambda_{n} T} \int_{0}^{T} \int_{\partial D} u^{T}(t, x) \mathrm{d} \Gamma_{x} \mathrm{~d} t \leqslant \frac{\mathrm{y}_{n}^{1}-e^{-\lambda_{n} T} \mathrm{y}_{n}^{0}}{-\alpha_{n}} \leqslant \int_{0}^{T} \int_{\partial D} u^{T}(t, x) \mathrm{d} \Gamma_{x} \mathrm{~d} t
$$


that is,

$$
\frac{\mathrm{y}_{n}^{1}-e^{-\lambda_{n} T} \mathrm{y}_{n}^{0}}{-\alpha_{n}} \leqslant \int_{0}^{T} \int_{\partial D} u^{T}(t, x) \mathrm{d} \Gamma_{x} \mathrm{~d} t \leqslant \frac{e^{\lambda_{n} T} \mathrm{y}_{n}^{1}-\mathrm{y}_{n}^{0}}{-\alpha_{n}}
$$

Hence, if for every $T>0$ such a nonnegative control $u^{T}$ exists, then

$$
\lim _{T \rightarrow 0} \int_{0}^{T} \int_{\partial D} u^{T}(t, x) \mathrm{d} \Gamma_{x} \mathrm{~d} t=\frac{\mathrm{y}_{n}^{1}-\mathrm{y}_{n}^{0}}{-\alpha_{n}} \quad \forall n \in \mathbb{N}^{*}
$$

But this limit (denoted by $\gamma$ ) must be independent of $n$. Consequently, $\mathrm{y}_{n}^{0}$ must satisfy

$$
\mathrm{y}_{n}^{0}=\mathrm{y}_{n}^{1}+\alpha_{n} \gamma \quad\left(n \in \mathbb{N}^{*}\right) .
$$

Since $\mathrm{y}^{0} \in L^{2}(D)$, we also have,

$$
\sum_{n=1}^{\infty} \frac{\left|\mathrm{y}_{n}^{0}\right|^{2}}{\left\|\varphi_{n}\right\|_{L^{2}(D)}^{2}}<\infty
$$

But since,

$$
\begin{aligned}
\int_{D}\left|\varphi_{n}(x)\right|^{2} \mathrm{~d} x & =\omega_{d-1} \int_{0}^{1}\left|p_{n}(r)\right|^{2} r^{d-1} \mathrm{~d} r \\
& =\frac{-\omega_{d-1}}{\lambda_{n}} \int_{0}^{1} p_{n}(r) \frac{\mathrm{d}}{\mathrm{d} r}\left(r^{d-1} p_{n}^{\prime}(r)\right) \mathrm{d} r \\
& =\frac{\omega_{d-1}}{\lambda_{n}} \int_{0}^{1}\left|p_{n}^{\prime}(r)\right|^{2} r^{d-1} \mathrm{~d} r \\
& =\frac{-\omega_{d-1}}{(d-1) \lambda_{n}} \int_{0}^{1} p_{n}^{\prime}(r)\left(\lambda_{n} p_{n}(r)+p_{n}^{\prime \prime}(r)\right) r^{d} \mathrm{~d} r \\
& =\frac{-\omega_{d-1}}{(d-1)} \int_{0}^{1} p_{n}^{\prime}(r) p_{n}(r) r^{d} \mathrm{~d} r-\frac{\omega_{d-1}}{(d-1) \lambda_{n}} \int_{0}^{1} p_{n}^{\prime}(r) p_{n}^{\prime \prime}(r) r^{d} \mathrm{~d} r
\end{aligned}
$$

where $\omega_{d-1}=\int_{\partial D} \mathrm{~d} \Gamma_{x}$, and since,

$$
\begin{aligned}
\int_{0}^{1} p_{n}^{\prime}(r) p_{n}(r) r^{d} \mathrm{~d} r=\frac{1}{2} \int_{0}^{1} p_{n}^{\prime}(r) p_{n}(r) r^{d} \mathrm{~d} r-\frac{1}{2} \int_{0}^{1} p_{n}(r) \frac{\mathrm{d}}{\mathrm{d} r} & \left(r^{d} p_{n}(r)\right) \mathrm{d} r \\
& =-\frac{d}{2} \int_{0}^{1}\left|p_{n}(r)\right|^{2} r^{d-1} \mathrm{~d} r
\end{aligned}
$$

and

$$
\begin{aligned}
\int_{0}^{1} p_{n}^{\prime}(r) p_{n}^{\prime \prime}(r) r^{d} \mathrm{~d} r=\frac{1}{2} \int_{0}^{1} p_{n}^{\prime}(r) p_{n}^{\prime \prime}(r) r^{d} \mathrm{~d} r-\frac{1}{2} \int_{0}^{1} p_{n}^{\prime}(r) \frac{\mathrm{d}}{\mathrm{d} r}\left(r^{d} p_{n}^{\prime}(r)\right) \mathrm{d} r+\frac{\alpha_{n}^{2}}{2} \\
=-\frac{d}{2} \int_{0}^{1}\left|p_{n}^{\prime}(r)\right|^{2} r^{d-1} \mathrm{~d} r+\frac{\alpha_{n}^{2}}{2}
\end{aligned}
$$

(18c) together with the above equalities and (18a)-(18b) leads to

$$
\int_{D}\left|\varphi_{n}(x)\right|^{2} \mathrm{~d} x=\frac{d}{d-1} \int_{D}\left|\varphi_{n}(x)\right|^{2} \mathrm{~d} x-\frac{\omega_{d-1}}{(d-1) \lambda_{n}} \frac{\alpha_{n}^{2}}{2} .
$$

Consequently, $\left\|\varphi_{n}(x)\right\|_{L^{2}(D)}^{2}=\frac{\omega_{d-1}}{2 \lambda_{n}} \alpha_{n}^{2}$. Now combining (16) and (17) together with this equality, we find

$$
\sum_{n=1}^{+\infty} \frac{\left|\mathrm{y}_{n}^{1}\right|^{2}}{\left\|\varphi_{n}\right\|_{L^{2}(D)}}+\frac{2 \gamma}{\omega_{d-1}} \sum_{n=1}^{+\infty}\left(2 \frac{\mathrm{y}_{n}^{1}}{\alpha_{n}}+\gamma\right)^{2} \lambda_{n}<+\infty .
$$

The first sum is finite since $\mathrm{y}^{1} \in L^{2}(D)$ and if $\gamma \neq 0$ the second sum can be finite only if $\lim _{n \rightarrow \infty} \lambda_{n}\left(y_{n}^{1}+\alpha_{n} \frac{\gamma}{2}\right)=0$. But, we have,

$$
\lambda_{n} \mathrm{y}_{n}^{1}=\lambda_{n} \int_{\Omega} \mathrm{y}^{1}(x) \varphi_{n}(x) \mathrm{d} x=-\int_{\Omega} \mathrm{y}^{1}(x) \Delta \varphi_{n}(x) \mathrm{d} x=-\alpha_{n} \int_{\partial \Omega} \mathrm{y}^{1}(x) \mathrm{d} \Gamma_{x}
$$


and hence,

$$
\lambda_{n}\left(\mathrm{y}_{n}^{1}+\alpha_{n} \frac{\gamma}{2}\right)=\lambda_{n} \frac{\gamma}{2}-\int_{\partial D} \mathrm{y}^{1}(x) \mathrm{d} \Gamma_{x} \quad\left(n \in \mathbb{N}^{*}\right)
$$

Consequently, unless we have $\gamma=0$, we cannot have $\lim _{n \rightarrow \infty} \lambda_{n}\left(\mathrm{y}_{n}^{1}+\alpha_{n} \frac{\gamma}{2}\right)=0$.

All in all, we have proved that if for every $T>0$, there exists a nonnegative control $u^{T} \in L^{1}((0, T) \times \partial D)$ steering $\mathrm{y}^{0}$ to $\mathrm{y}^{1}$ in time $T$, then we have, $\lim _{T \rightarrow 0} \int_{0}^{T} \int_{\partial D} u(t, x) \mathrm{d} \Gamma_{x} \mathrm{~d} t=0$.

Let us now prove that, if for every $T>0$ there exists a nonnegative control $u^{T} \in L^{1}((0, T) \times$ $\partial D)$ steering the solution $y$ of (11) from $\mathrm{y}^{0}$ to $\mathrm{y}^{1}$, then we must have $\mathrm{y}^{0} \equiv \mathrm{y}^{1}$.

Let $\varphi$ be an eigenfunction of the Dirichlet-Laplacian operator, with eigenvalue $\lambda>0$, i.e., $\Delta \varphi=-\lambda \varphi$ on $D$ and $\varphi=0$ on $\partial D$. It is well known that any such eigenfunction is smooth and that there exists an orthonormal basis of $L^{2}(D)$ consisting of such eigenfunctions. As for (14), we obtain

$$
\int_{D} \mathrm{y}^{1}(x) \varphi(x) \mathrm{d} x-e^{-\lambda T} \int_{D} \mathrm{y}^{0}(x) \varphi(x) \mathrm{d} x=-\int_{0}^{T} e^{-\lambda(T-t)} \int_{D} \nabla \varphi(x) \cdot \mathrm{n}(x) u^{T}(t, x) \mathrm{d} \Gamma_{x} \mathrm{~d} t .
$$

But since $\varphi$ is smooth and since $\int_{0}^{T} \int_{D} u^{T}(t, x) \mathrm{d} \Gamma_{x} \mathrm{~d} t=\int_{0}^{T} \int_{D}\left|u^{T}(t, x)\right| \mathrm{d} \Gamma_{x} \mathrm{~d} t \rightarrow 0$ as $T \rightarrow 0$, we conclude that

$$
\begin{aligned}
0= & -\lim _{T \rightarrow 0} \int_{0}^{T} e^{-\lambda(T-t)} \int_{D} \nabla \varphi(x) \cdot \mathrm{n}(x) u^{T}(t, x) \mathrm{d} \Gamma_{x} \mathrm{~d} t \\
& =\lim _{T \rightarrow 0}\left(\mathrm{y}^{1} \int_{D} \varphi(x) \mathrm{d} x-e^{-\lambda T} \int_{D} \mathrm{y}^{0}(x) \varphi(x) \mathrm{d} x\right)=\int_{D} \mathrm{y}^{1}(x) \varphi(x) \mathrm{d} x-\int_{D} \mathrm{y}^{0}(x) \varphi(x) \mathrm{d} x .
\end{aligned}
$$

This means that $\mathrm{y}^{1}$ and $\mathrm{y}^{0}$ have the same $L^{2}$-projections on any eigenfunction of the DirichletLaplacian operator, and thus they do coincide. This shows that $\underline{T}\left(\mathrm{y}^{0}, \mathrm{y}^{1}\right)$ is positive.

Set $\underline{T}=\underline{T}\left(\mathrm{y}^{0}, \mathrm{y}^{1}\right)$. Let us now prove the existence of a control in $\mathcal{M}((0, \underline{T}) \partial D)$. First of all, for every $n \in \mathbb{N}^{*}$, there exist a nonnegative control $u^{n} \in L^{1}\left(\left(0, \underline{T}+\frac{1}{n}\right) \times \partial D\right)$ steering $y$, solution of (11), from $\mathrm{y}^{0}$ to $\mathrm{y}^{1}$ in time $\underline{T}+\frac{1}{n}$. According to (15), this control satisfies

$$
\left\|u^{n}\right\|_{L^{1}\left(\left(0, \underline{T}+\frac{1}{n}\right) \times \partial D\right)} \leqslant \frac{e^{\lambda_{1}\left(\underline{T}+\frac{1}{n}\right)} \mathrm{y}_{1}^{1}-\mathrm{y}_{1}^{0}}{-\alpha_{1}} \leqslant \frac{e^{\lambda_{1}(\underline{T}+1)}\left|\mathrm{y}_{1}^{1}\right|+\left|\mathrm{y}_{1}^{0}\right|}{\left|\alpha_{1}\right|}
$$

Then, the sequence $\left(u^{n}\right)_{n}$ is bounded in $L^{1}(0, \underline{T}+1)$ and therefore, by weak compactness of $\mathcal{M}(0, \underline{T})$, up to a subsequence it converges in the weak sense of Radon measures to some control $\underline{u}$ in $\mathcal{M}(0, \underline{T})$.

Clearly, the limit controls satisfy the nonnegativity constraint and the limit solution solves the limit non-homogeneous Dirichlet problem (11) in the sense of transposition and the limit solution reaches the target $\mathrm{y}^{1}$ in time $\underline{T}$.

Remark 5. In the proof of Theorem 3, proving that $\underline{T}\left(\mathrm{y}^{0}, \mathrm{y}^{1}\right)>0$ (unless $\left.\mathrm{y}^{0}=\mathrm{y}^{1}\right)$ did not require that $\mathrm{y}^{1}$ is a steady-state. In fact we always have $\underline{T}\left(\mathrm{y}^{0}, \mathrm{y}^{1}\right)>0$ if $\mathrm{y}^{0} \neq \mathrm{y}^{1}$, with the convention that $\underline{T}\left(\mathrm{y}^{0}, \mathrm{y}^{1}\right)=+\infty$ if $\mathrm{y}^{1}$ is not reachable from $\mathrm{y}^{0}$.

As a consequence of the proof, and in particular of (15), we get the following lower bound for $\underline{T}\left(\mathrm{y}^{0}, \mathrm{y}^{1}\right)$, in the case where $\mathrm{y}^{0}$ is constant.

Corollary 1. Let $\mathrm{y}^{0} \in \mathbb{R}$ and $\mathrm{y}^{1} \in(0,+\infty)$ be arbitrary. Then the minimal time $\underline{T}=\underline{T}\left(\mathrm{y}^{0}, \mathrm{y}^{1}\right)$ is such that

$$
\sup _{n \in \mathbb{N}^{*}}\left(\frac{\mathrm{y}^{1}}{\lambda_{n}}-\frac{e^{-\lambda_{n} \underline{T}} \mathrm{y}^{0}}{\lambda_{n}}\right) \leqslant \inf _{n \in \mathbb{N}^{*}}\left(\frac{e^{\lambda_{n} \underline{T}} \mathrm{y}^{1}}{\lambda_{n}}-\frac{\mathrm{y}^{0}}{\lambda_{n}}\right) \quad \text { and } \quad 0 \leqslant \inf _{n \in \mathbb{N}^{*}}\left(\frac{e^{\lambda_{n} \underline{T}} \mathrm{y}^{1}}{\lambda_{n}}-\frac{\mathrm{y}^{0}}{\lambda_{n}}\right),
$$

where $\left(\lambda_{n}\right)_{n \in \mathbb{N}^{*}}$ is defined by the Sturm-Liouville problem (13). 


\subsection{General domain and nonnegativity state constraint}

Let $\Omega$ be a bounded and smooth domain $\mathbb{R}^{d}$ of class $C^{2}$ and consider the control problem

$$
\begin{aligned}
\partial_{t} y(t, x) & =\Delta y(t, x) & & (t>0, x \in \Omega), \\
y(t, x) & =u(t, x) & & (t>0, x \in \partial \Omega),
\end{aligned}
$$

with nonnegative initial condition $\mathrm{y}^{0} \in L^{2}(\Omega)$,

$$
y(0, x)=\mathrm{y}^{0}(x) \geqslant 0 \quad(x \in \Omega) .
$$

The system is well known to be null-controllable in any time $T>0$ with controls $u \in L^{2}((0, T) \times$ $\partial \Omega$ ) (see $[7,12,28])$. The question we analyze is whether controllability is true as well under the additional nonnegativity requirement on the state

$$
y(t, x) \geqslant 0, \quad t>0, x \in \Omega,
$$

and whether this state constraint causes a positive minimal time (or waiting time).

Remark 6. Here again, as in Remark 4, by the comparison principle, the nonnegativity state constraint $y \geqslant 0$ is equivalent to the nonnegativity control constraint $u \geqslant 0$.

Theorem 4. Let $\mathrm{y}^{0} \in L^{2}(\Omega)$ be such that $\mathrm{y}^{0} \geqslant 0$, and let $\mathrm{y}^{1} \in L^{2}(\Omega)$ be a steady-state of (19). We assume that $\mathrm{y}^{0} \neq \mathrm{y}^{1}$ and that there exists $\varepsilon>0$ such that $\mathrm{y}^{1}(x) \geqslant \varepsilon$ for every $x \in \Omega$. Then:

- There exist $T>0$ (large enough) and a control $u \in L^{2}((0, T) \times \partial \Omega)$ such that the corresponding solution of $(19)$, with $y(0)=\mathrm{y}^{0}$, satisfies the state constraint $(21)$ and reaches $y(T)=\mathrm{y}^{1}$.

- Defining

$$
\underline{T}\left(\mathrm{y}^{0}, \mathrm{y}^{1}\right)=\inf \left\{T>0, \exists u \in L^{1}\left((0, T) \times \Gamma_{0}\right) \text { s.t. } y(t, x) \geqslant 0 \text { and } y(T, \cdot)=\mathrm{y}^{1}\right\},
$$

we have $\underline{T}\left(\mathrm{y}^{0}, \mathrm{y}^{1}\right)>0$.

- For $T=\underline{T}\left(\mathrm{y}^{0}, \mathrm{y}^{1}\right)$, there exists a control $\underline{u} \in \mathcal{M}((0, T) \times \partial \Omega)$ (the space of Radon measures) steering the heat equation (19) from $\mathrm{y}^{\overline{0}}$ to $\mathrm{y}^{1}$ in time $T$ under the nonnegativity state constraint (21).

Remark 7. Proceeding as in the proof of Proposition 1, one can show that for every $\tau>0$, there exists a control in time $\underline{T}\left(\mathrm{y}^{0}, \mathrm{y}^{1}\right)+\tau$ with arbitrary regularity. However, there does not seem to exist a systematic way of proving that controls for the heat equation have arbitrary regularity.

Proof of Theorem 4. The proof of constrained controllability in large time is the same as in 1D. We give a sketch. Working on the shifted state $z=y-\mathrm{y}^{1}$, it is sufficient to address the problem of controlling the system to the zero final state. Then, using the fact that the cost of controlling the system decreases exponentially as $T \rightarrow+\infty$, and that, by regularity considerations, the controls can be taken in $L^{\infty}$, we conclude that, for $T$ large enough, the $L^{\infty}$-norm of the control $v$ driving $z$ to zero in time $T$ is smaller than ${ }^{1}$. The control $u$ for the original state $y$ is then $u=v+\left.\mathrm{y}^{1}\right|_{\partial \Omega}$, which can then be guaranteed to be nonnegative.

The positivity of the minimal time is established by comparison, based on the result of the previous subsection in the case where $\Omega$ is a ball. Indeed, let $D$ be the largest ball contained in $\Omega$ and such that $\left.\mathrm{y}^{0}\right|_{D} \neq\left.\mathrm{y}^{1}\right|_{D}$. Assume that the heat equation (19) is controllable in time $T$ under the positivity constraint. Let $z$ be equal to the restriction of $y$ to $D$, and let $v$ be the restriction of $y$ to $\partial D$. Then, obviously, $v$ is a control for $z$ in the ball $D$, preserving the control constraint. This immediately implies that the control time $T$ has to satisfy the lower bounds of the previous section. Of course, this argument applies to any ball $D$ included in $\Omega$ and, as indicated in the remark hereafter, is also valid for other boundary conditions. 
The proof of the existence of a nonnegative control $\mathcal{M}\left(\left(0, \underline{T}\left(\mathrm{y}^{0}, \mathrm{y}^{1}\right)\right) \times \partial \Omega\right)$ is similar to the one of Theorem 3. More precisely, denoting by $\lambda_{0}$ the first eigenvalue of the DirichletLaplacian operator and by $\varphi_{0}$ the corresponding normalized eigenvector, and defining $y_{0}(t)=$ $\int_{\Omega} y(t, x) \varphi_{0}(x) \mathrm{d} x$, we have $\dot{y}_{0}(t)=-\int_{\partial \Omega} u(t, x) \nabla \varphi_{0}(x) \cdot \mathrm{n}(x) \mathrm{d} \Gamma_{x}-\lambda_{0} y_{0}(t)$, and hence,

$$
-\int_{0}^{T} e^{-\lambda_{0}(T-t)} \int_{\partial \Omega} u(t, x) \nabla \varphi_{0}(x) \cdot \mathrm{n}(x) \mathrm{d} \Gamma_{x} \mathrm{~d} t=y_{0}(T)-e^{-\lambda_{0} T} y_{0}(0) .
$$

It is well known that $\varphi_{0}$ keeps a constant sign, which can be chosen to be positive, and that its normal derivative does not vanish. Therefore $\inf _{\partial \Omega}\left(-\nabla \varphi_{0} \cdot \mathrm{n}\right)=\alpha_{0}>0$, and hence

$$
\int_{0}^{T} \int_{\partial \Omega} u(t, x) \mathrm{d} \Gamma_{x} \mathrm{~d} t \leqslant \frac{1}{\alpha}\left(e^{\lambda_{0} T} \mathrm{y}_{0}^{1}-\mathrm{y}_{0}^{0}\right)
$$

with $\mathrm{y}_{0}^{i}=\int_{\Omega} \mathrm{y}^{i}(x) \varphi_{0}(x) \mathrm{d} x$ for $i=0,1$. This ensures that any nonnegative control is bounded in $L^{1}$ norm. We conclude as in the proof of Theorem 3.

Several remarks are in order.

Remark 8. We consider the case where the control acts only on a proper subset $\Gamma_{0} \subset \partial \Omega$,

$$
\begin{aligned}
\partial_{t} y(t, x) & =\Delta y(t, x) & & (t>0, x \in \Omega), \\
y(t, x) & =u(t, x) & & \left(t>0, x \in \Gamma_{0}\right), \\
y(t, x) & =0 & & (t>0, x \in \partial \Omega \\
y(0, x) & =\mathrm{y}^{0}(x) \geqslant 0 & & (x \in \Omega) .
\end{aligned}
$$

Given a target $\mathrm{y}^{1} \in(0,+\infty)$ that is assumed to be reachable from $\mathrm{y}^{0}$ (in sufficiently large time) with nonnegative controls, we have $\underline{T}\left(\mathrm{y}^{0}, \mathrm{y}^{1}\right)>0$. This follows, similarly, from a localization argument in a ball contained in $\Omega$, combined with Theorem 3 and Remark 5 .

The main difficulty here is to establish reachability in large enough time. The question is delicate because the constant $\mathrm{y}^{1}>0$ is not a steady-state of $(22)$. Actually, reachability is even not clear even if we assume that $\mathrm{y}^{1}$ is a nonnegative steady-state, i.e., satisfying $\Delta y_{1}(x)=0$ in $\Omega, y_{1}=\bar{u}$ on $\Gamma_{0}$ and $y_{1}=0$ on $\partial \Omega \backslash \Gamma_{0}$, for some nonnegative $\bar{u} \in L^{2}\left(\Gamma_{0}\right)$.

Following [18, Theorem 4.1 (b)], it is possible to prove that, for every $\varepsilon>0$, there exist $T>0$ and a control $u \in L^{2}\left((0, T) \times \Gamma_{0}\right)$, satisfying $u \geqslant-\varepsilon$ for some $\varepsilon>0$, steering the solution of (22) from $\mathrm{y}^{0}$ to $\mathrm{y}^{1}$ in time $T$. But it is not clear if one can realize it with $\varepsilon=0$. In fact, in the particular case where $\bar{u}=0$, i.e., when $\mathrm{y}^{1}=0$, due to the comparison principle, it is not possible to steer any $\mathrm{y}^{0} \geqslant 0$, with $\mathrm{y}^{0} \in L^{2}(\Omega) \backslash\{0\}$ to 0 .

Remark 9. The result can be extended to more general elliptic constant coefficient operators, not necessarily coinciding with the Laplacian. They hold in particular for more general parabolic problems of the form

$$
\partial_{t} y=\operatorname{div}(A \nabla y)
$$

where $A \in \mathbb{R}^{d \times d}$ is a constant coefficient positive matrix.

Indeed, there exists an orthogonal matrix $P \in \mathbb{R}^{d \times d}$ such that $A=P P^{\top}$. Setting $\tilde{x}=P x$ and $\tilde{y}(t, \tilde{x})=y(t, x)$, the parabolic problem above reduces to $\partial_{t} \tilde{y}(t, \tilde{x})=\frac{1}{|P|} \Delta \tilde{y}(t, \tilde{x})$.

Remark 10. The positivity of the minimal time follows from the comparison with the Dirichlet control problem with nonnegativity control constraints over the largest ball included in $\Omega$. This leads to lower bounds on the minimal time.

Remark 11. The minimal time is the same both for nonnegativity constraints on the state and on the control. This can be easily seen by the comparison principle since solutions with nonnegative initial data and Dirichlet controls are nonnegative everywhere. Similarly, if the solution itself is nonnegative, of course, the Dirichlet controls, which are simply the restriction to the boundary of the state are nonnegative as well. 
Remark 12. The same result is valid for other boundary conditions, for instance Neumann, provided that we deal with nonnegativity state constraints. This is the aim of Section 4.2. But, as we shall see in Section 4.4, dealing with constrained Neumann controls leads to different results.

Remark 13. Similar results hold when the control is acting in some interior subdomain $\omega$ of $\Omega$, i.e., for the model

$$
\partial_{t} y(t, x)=\Delta y(t, x)+u(t, x) \mathbf{1}_{\omega}(x),
$$

under any boundary conditions ensuring that constants states are steady-states. Here $u=$ $u(t, x)$ is the control and $\mathbf{1}_{\omega}$ is the characteristic function of the subset $\omega$ where the control is applied. The proof relies again on comparison arguments. Here, it suffices to consider a ball $D \subset \Omega \backslash \omega$ and to apply the arguments above; the action of the external force $u$ applied in $\omega$ is not seen anymore. This is developed with more details in Section 4.3.

\subsection{Relationship with the viscous Hamilton-Jacobi equation}

As observed by M. Tucsnak in [24], the waiting time phenomenon for the control under nonnegativity state constraints of the linear heat equation is related to that on the control of the viscous Hamilton-Jacobi equations developed in [17].

Indeed, let us consider the Dirichlet boundary control problem for the heat equation under the state constraint $y(t, x) \geqslant 0$. The logarithmic change of variable $z(t, x)=-\ln y(t, x)$ transforms the linear heat equation into the viscous Hamilton-Jacobi equation

$$
\dot{z}-\Delta z+|\nabla z|^{2}=0 \quad(t>0, x \in \Omega)
$$

with the initial condition $z(0, x)=-\ln \mathrm{y}^{0}(x)$ and constant target state $\mathrm{z}^{1}=-\ln \mathrm{y}^{1}$.

The null controllability of (23), with interior control localized in a subset $\omega$ of the domain $\Omega$ and Dirichlet boundary conditions, has been studied in [17]. More precisely, in [17, Theorem 1.1], the authors prove that any initial condition $\mathrm{z}^{0}$ for $(23)$ can be steered to 0 is any time $T$ larger than some time $T_{*}$ depending only on $\left\|z^{0}\right\|_{L^{\infty}(\Omega)}$. This result does not ensure that there always exists a positive minimal time for every initial condition $z^{0}$, but it ensures that there exists a function for which there exists a waiting time. It would be interesting to compare the lower bounds on the waiting time for these typical functions obtained in the present paper directly for the linear heat equation with those in [17]. But they are, in some sense, of the same nature since the application of the logarithmic change of variables to the first eigenfunction of the Dirichlet Laplacian leads to the classical barrier functions for elliptic viscous Hamilton-Jacobi equations that are used in [17] to establish the existence of a waiting time for the viscous Hamilton-Jacobi equation.

Our results in Section 4 show the existence of a waiting time whatever the initial nonnegative initial condition $\mathrm{y}^{0} \neq \mathrm{y}^{1}$ is. This leads to the existence of a positive minimal time for the controllability of $z$, taking into account that $y(t, x)=\exp (-z(t, x))$. Of course this argument, which allows one to compare the control of the linear heat equation and that of the viscous Hamilton-Jacobi equation with the logarithmic change of variables, can only be applied under the condition $y \geqslant 0$.

\section{Generalizations}

\subsection{Mixed Dirichlet-Neumann boundary conditions}

In $1 \mathrm{D}$, the symmetry properties of the controlled solutions allow to obtain some particular results for mixed boundary conditions of Dirichlet-Neumann type.

Going back to Theorem 2 and proceeding with symmetry considerations, when $\mathrm{y}^{0}$ is symmetric with respect to $x=1 / 2$ and, in particular, when it is a constant state, we can take 
$u_{0}=u_{1}$. Then $u_{0}$ can also be viewed as the only boundary control for the mixed DirichletNeumann system

$$
\begin{aligned}
\partial_{t} y(t, x) & =\partial_{x}^{2} y(t, x) & & \text { in }(0, T) \times(0,1 / 2), \\
\partial_{x} y\left(t, \frac{1}{2}\right) & =0 & & (t \in(0, T)), \\
y(t, 0) & =u_{0}(t) & & (t \in(0, T)), \\
y(0, x) & =\mathrm{y}^{0} & & (x \in(0,1))
\end{aligned}
$$

steering the solution from $\mathrm{y}^{0}$ to $\mathrm{y}^{1}$ in time $T$.

Note that in this system the control enters through the Dirichlet boundary condition at the left boundary $x=0$, while the homogeneous Neumann boundary condition is satisfied at the right boundary $x=1 / 2$.

The converse result holds as well. Indeed, the even (with respect to $x=1 / 2$ ) extension of the solution of $(24)$ solves the Dirichlet control problem in $(0,1)$ with equal controls at both extremities $x=0$ and $x=1$. Consequently, the time required to steer the solution of (1), with control constraints (5), from $\mathrm{y}^{0}$ to $\mathrm{y}^{1}$, coincides with the one required to steer $(24)$, with the control constraint $u_{0}(t) \geqslant 0$, from $\mathrm{y}^{0}$ to $\mathrm{y}^{1}$.

Similar symmetry considerations can also be developed in the multi-dimensional case when the domain $\Omega$ under consideration enjoys adequate symmetry properties. This allows one to relate the Dirichlet control problem in the full domain $\Omega$ to the mixed Dirichlet-Neumann problem in some subdomains. This can be applied, for instance, when the domain $\Omega$ is a square, linking the Dirichlet control problem in $\Omega$ to the mixed control problem in the rectangle corresponding to half of $\Omega$.

\subsection{Neumann control under nonnegativity state constraints}

Let $\Omega$ be a bounded domain of $\mathbb{R}^{d}$ with $C^{2}$ boundary.

We first consider the heat equation with Neumann boundary conditions

$$
\begin{aligned}
& \partial_{t} y(t, x)=\Delta y(t, x) \\
& (t>0, x \in \Omega), \\
& \partial_{\nu} y(t, x)=v(t, x) \\
& (t>0, x \in \partial \Omega) \text {, }
\end{aligned}
$$

with initial condition $y(0, x)=\mathrm{y}^{0} \in L^{2}(\Omega)$, with $\mathrm{y}^{0} \geqslant 0$. Note that, here, the Neumann control acts along the whole boundary. We consider the problem of reaching the constant target state $\mathrm{y}^{1}>0\left(\right.$ with $\mathrm{y}^{0} \neq \mathrm{y}^{1}$ ) under the nonnegativity state constraint (21).

Controllability, without taking into account any constraint, has been established in [19]. We claim that controllability under the nonnegativity state constraint can be achieved in time sufficiently large, and that the minimal time for this constrained control problem is positive. Actually, the minimal times under state constraints both for Dirichlet and Neumann control problems coincide with the minimal time under nonnegativity constraints on the Dirichlet control. Indeed, the Neumann control $v$ can be taken to be the trace on $\partial \Omega$ of the normal derivative of the controlled trajectory by means of Dirichlet controls, under state constraints.

Let us now consider the more general situation where the Neumann control acts only on a proper subset $\Gamma_{0} \subset \partial \Omega$ of the boundary:

$$
\begin{aligned}
\partial_{t} y(t, x) & =\Delta y(t, x) & & (t>0, x \in \Omega), \\
\partial_{\nu} y(t, x) & =v(t, x) & & \left(t>0, x \in \Gamma_{0}\right), \\
\partial_{\nu} y(t, x) & =0 & & \left(t>0, x \in \partial \Omega \backslash \Gamma_{0}\right), \\
y(0, x) & =\mathrm{y}^{0}(x) & & (x \in \Omega) .
\end{aligned}
$$

Theorem 5. Let $\mathrm{y}^{0} \in L^{2}(\Omega) \backslash\{0\}$ be such that $\mathrm{y}^{0}(x) \geqslant 0$ for every $x \in \Omega$, and let $\mathrm{y}^{1} \in L^{2}(\Omega)$ be a steady-state of (26). We assume that there exists $\varepsilon>0$ such that $\mathrm{y}^{1}(x) \geqslant \varepsilon$ for every $x \in \Omega$. Then: 
- There exist $T>0$ and a control $v \in L^{2}\left((0, T) \times \Gamma_{0}\right)$ such that the corresponding solution $y$ of (26) with initial condition $y(0)=\mathrm{y}^{0}$ satisfies $y(T)=\mathrm{y}^{1}$ and $y(t, x) \geqslant 0$ for all $(t, x) \in(0, T) \times \Omega$.

- Defining

$$
\underline{T}\left(\mathrm{y}^{0}, \mathrm{y}^{1}\right)=\inf \left\{T>0, \exists v \in L^{1}\left((0, T) \times \Gamma_{0}\right) \text { s.t. } y(t, x) \geqslant 0 \text { and } y(T)=\mathrm{y}^{1}\right\},
$$

we have $\underline{T}\left(\mathrm{y}^{0}, \mathrm{y}^{1}\right)>0$ whenever $\mathrm{y}^{0} \neq \mathrm{y}^{1}$.

Remark 14. In the above definition of $\underline{T}\left(\mathrm{y}^{0}, \mathrm{y}^{1}\right)$ we take controls in $L^{1}$, in order to have a large class of controls (see also Remark 15 further). We are not able to prove the existence of a Radon measure control $u$ realizing the controllability exactly in time $\underline{T}\left(\mathrm{y}^{0}, \mathrm{y}^{1}\right)$.

Proof. The previous argument does not apply directly. First, the positivity of the minimal control time is established similarly: indeed, if the solution of the control problem (26) satisfies the nonnegativity state constraint, then it can also be viewed as a controlled trajectory with nonnegative Dirichlet controls. But the fact that the system can be controlled while preserving the nonnegativity of the state in large time requires further analysis. This goes as follows. Recall that, for the Dirichlet control problem, the property of constrained controllability was established by using the fact that the cost of controlling the system tends to zero exponentially as $T \rightarrow+\infty$. This argument does not apply directly in the context of Neumann boundary controls. In fact the existence of nontrivial steady-states for the adjoint Neumann problem

$$
\begin{aligned}
-\partial_{t} \varphi(t, x) & =\Delta \varphi(t, x) & & (t \in(0, T), x \in \Omega), \\
\partial_{\nu} \varphi(t, x) & =0 & & (t \in(0, T), x \in \partial \Omega), \\
\varphi(T, x) & =\varphi^{T} & & (x \in \Omega),
\end{aligned}
$$

is an impediment for the observability constant to decay exponentially. The relevant observability constant in the present setting, which is

$$
\|\varphi(0)\|_{L^{2}(\Omega)}^{2} \leqslant C(T) \int_{0}^{T} \int_{\Gamma_{0}} \varphi^{2} \mathrm{~d} \sigma \mathrm{d} t
$$

is well known to be satisfied for any $T>0$ (see [19]). But the existence of trivial constant solutions $\varphi \equiv 1$ prevents the observability constant $C(T)$ from decaying exponentially. It is however easy to see that $C(T)$ decays as $\mathrm{O}(1 / T)$ when $T \rightarrow+\infty$. This is so because, once the existence of an observability constant $C^{*}$ is established for some specific value of $T=T^{*}$, the observability constant $C(T)$ can be guaranteed to be of the order of $C^{*} / k$ for $T=k T^{*}$.

This also ensures that one can steer the system (26) to 0 in time $T$ with a control $u \epsilon$ $L^{2}\left((0, T) \times \Gamma_{0}\right)$ such that

$$
\|u\|_{L^{2}\left((0, T) \times \Gamma_{0}\right)}^{2} \leqslant C(T)\left\|\mathrm{y}^{0}\right\|_{L^{2}(\Omega)}^{2} .
$$

Similar results hold when the observation is taken in a weaker space. This leads in particular to the existence of a control $u \in L^{2}\left((0, T) ; H^{r}\left(\Gamma_{0}\right)\right) \cap H^{s}\left((0, T) ; L^{2}\left(\Gamma_{0}\right)\right)$, for any given $r, s \geqslant 0$, satisfying

$$
\|u\|_{L^{2}\left((0, T) ; H^{r}\left(\Gamma_{0}\right)\right)}^{2}+\|u\|_{H^{s}\left((0, T) ; L^{2}\left(\Gamma_{0}\right)\right)}^{2} \leqslant C_{r, s}(T)\left\|\mathrm{y}^{0}\right\|_{L^{2}(\Omega)}^{2} \quad(T>0),
$$

steering (26) to 0 in time $T$.

Furthermore, in view of $\left[13, \S 13.1\right.$ and Theorem 2.1], for $r$ and $s$ large enough and for $\mathrm{y}^{0}$ regular enough, the solution $y$ of (26) with initial condition $y(0)=\mathrm{y}^{0}$ satisfies $y(t, \cdot) \in L^{\infty}(\Omega)$, and due to well-posedness of the heat equation, there also exists $K(T)>0$, not depending on the control $u$, such that

$$
\left\|y(t)-y_{0}(t)\right\|_{L^{\infty}(\Omega)} \leqslant K(T)\left(\|u\|_{L^{2}\left((0, T) ; H^{r}\left(\Gamma_{0}\right)\right)}+\|u\|_{H^{s}\left((0, T) ; L^{2}\left(\Gamma_{0}\right)\right)}\right) \quad(t \in(0, T)),
$$

where $y_{0}$ is the solution of (26) with control $u=0$ and initial condition $\mathrm{y}^{0}$. All in all, we have obtained the following local controllability result. 
Lemma 3. Let $\mathrm{y}^{0} \in H^{1}(\Omega)$ and let $\tau>0$ be arbitrary. There exist $\kappa(T)>0$ and a control $u \in L^{2}\left((0, T) \times \Gamma_{0}\right)$ such that the solution $y$ of $(26)$ with initial condition $y(0)=\mathrm{y}^{0}$ and control $u$ satisfies $y(T)=0$ and $\left\|y(t)-y_{0}(t)\right\|_{L^{\infty}(\Omega)} \leqslant \kappa(T)\left\|\mathrm{y}^{0}\right\|_{L^{2}(\Omega)}$, where $y_{0}$ is solution of (26) with control $u=0$ and initial condition $\mathrm{y}^{0}$.

We are now in a position to prove the theorem. In order to avoid additional notations, we only present the case where $\mathrm{y}^{1}$ is a constant steady-state. The general case, can be proved by the same way. Let $\tau>0$ and let $\varepsilon>0$ be arbitrary. We will proceed in several steps:

1. We take a null control during a long time $T_{0}(\varepsilon)$ so that the solution $y$ of $(26)$ with null control and initial condition $y(0)=\mathrm{y}^{0}$ satisfies

$$
\left\|y\left(T_{0}(\varepsilon)\right)-\frac{1}{|\Omega|} \int_{\Omega} \mathrm{y}^{0}\right\|_{L^{\infty}(\Omega)}^{<\varepsilon} \quad \text { and } \quad\left\|y\left(T_{0}(\varepsilon)\right)-\frac{1}{|\Omega|} \int_{\Omega} \mathrm{y}^{0}\right\|_{L^{2}(\Omega)}<\varepsilon .
$$

We set $\mathrm{y}_{0}=y\left(T_{0}(\varepsilon)\right)$ and $\overline{\mathrm{y}}_{0}=\frac{1}{|\Omega|} \int_{\Omega} \mathrm{y}^{0}$.

2. We build a control $u_{0}$ steering (26) from the initial condition $y(0)=\mathrm{y}_{0}$ to the target $\overline{\mathrm{y}}_{0}$ in time $\tau$.

3. We define a finite sequence $\left(\overline{\mathrm{y}}_{k}\right)_{k=0, \ldots, K}$ of positive values such that $\overline{\mathrm{y}}_{K}=\mathrm{y}^{1}$ and $\mid \overline{\mathrm{y}}_{k+1}-$ $\overline{\mathrm{y}}_{k} \mid<\varepsilon$ for every $k \in\{0, \ldots, K-1\}$.

4. For $k \in\{1, \ldots, K\}$, we build a control $u_{k}$ steering (26) from the initial condition $y(0)=$ $\overline{\mathrm{y}}_{k-1}$ to the target $\overline{\mathrm{y}}_{k}$ in time $\tau$.

Once all this process is done, we check that there exists $\varepsilon=\varepsilon(\tau)>0$ small enough such that the solution $y$ with initial condition $y(0)=\mathrm{y}^{0}$ and with control

$u(t)=\left\{\begin{array}{ll}0 & \text { if } t \in\left(0, T_{0}(\varepsilon)\right), \\ u_{0}\left(t-T_{0}(\varepsilon)\right) & \text { if } t \in\left(T_{0}(\varepsilon), T_{0}(\varepsilon)+\tau\right), \\ \vdots & \\ u_{K}\left(t-T_{0}(\varepsilon)-K \tau\right) & \text { if } t \in\left(T_{0}(\varepsilon)+K \tau, T_{0}(\varepsilon)+(K+1) \tau\right),\end{array} \quad\left(t \in\left(0, T_{0}(\varepsilon)+(K+1) \tau\right)\right.\right.$

satisfies $y(t, x) \geqslant 0$ for all $(t, x) \in\left(0, T_{0}(\varepsilon)+(K+1) \tau\right) \times \Omega$.

Step 1. Using a spectral expansion, it is easy to see that the solution $y$ of (26) with null control and initial condition $y(0)=\mathrm{y}^{0}$ converges in any Sobolev norm to $\overline{\mathrm{y}}_{0}$ as $t \rightarrow+\infty$. Therefore, there exists $T_{0}(\varepsilon)>0$ such that $\| y\left(T_{0}(\varepsilon)-\bar{y}_{0} \|_{L^{\infty}(\Omega)}<\varepsilon\right.$ and $\| y\left(T_{0}(\varepsilon)-\bar{y}_{0} \|_{L^{2}(\Omega)}<\right.$ $\varepsilon$. Furthermore, since $\mathrm{y}^{0}$ is nonnegative and nontrivial, we have $\overline{\mathrm{y}}_{0}>0$ and $y(t, x) \geqslant 0$ on $\left(0, T_{0}(\varepsilon)\right) \times \Omega$. We set $\mathrm{y}_{0}=y\left(T_{0}(\varepsilon)\right) \in H^{1}(\Omega)$.

Step 2. According to Lemma 3, there exist $\kappa(\tau)>0$, only depending on $\tau$, and a control $u_{0} \in L^{2}\left((0, \tau) \times \Gamma_{0}\right)$ such that the solution $y$ of $(26)$ with initial condition $y(0)=\mathrm{y}_{0}$ and control $u_{0}$ satisfies $y(\tau)=\bar{y}_{0}$ and

$$
\left\|y(t)-y_{0}(t)\right\|_{L^{\infty}(\Omega)} \leqslant \kappa(\tau)\left\|\mathrm{y}_{0}-\overline{\mathrm{y}}_{0}\right\|_{L^{2}(\Omega)} \leqslant \varepsilon \kappa(\tau) \quad(t \in(0, \tau)),
$$

where $y_{0}$ is the solution of (26) with initial condition $\mathrm{y}_{0}$ and null control. Since $\| y_{0}(t)-$ $\overline{\mathrm{y}}_{0} \|_{L^{\infty}(\Omega)} \leqslant \varepsilon$, we easily obtain that $\left\|y(t)-\overline{\mathrm{y}}_{0}\right\|_{L^{\infty}(\Omega)} \leqslant \varepsilon(1+\kappa(\tau))$ for $t \in(0, \tau)$, i.e., $y(t, x) \geqslant$ $\overline{\mathrm{y}}_{0}-\varepsilon(1+\kappa(\tau))$ on $(0, \tau) \times \Omega$.

Step 3. Setting $K=K(\varepsilon) \geqslant\left|\mathrm{y}^{1}-\overline{\mathrm{y}}_{0}\right| / \varepsilon$ and $\overline{\mathrm{y}}_{k}=\frac{k}{K} \mathrm{y}^{1}+\left(1-\frac{k}{K}\right) \overline{\mathrm{y}}_{0}$ for $k \in\{0, \ldots, K\}$, we have $\left|\overline{\mathrm{y}}_{k+1}-\overline{\mathrm{y}}_{k}\right| \leqslant \varepsilon$ and $\overline{\mathrm{y}}_{K}=\mathrm{y}^{1}$. 
Step 4. Let $k \in\{1, \ldots, K(\varepsilon)\}$. According to Lemma 3, there exist $\kappa(\tau)>0$, only depending on $\tau$ (and independent of $k$, this constant is the same as in Step 2), and a control $u_{k} \in$ $L^{2}((0, \tau) \times \Gamma)$ such that the solution $y$ of $(26)$ with initial condition $y(0)=\mathrm{y}_{k-1}$ and control $u_{k}$ satisfies $y(\tau)=\bar{y}_{k}$ and

$$
\left\|y(t)-\overline{\mathrm{y}}_{k-1}\right\|_{L^{\infty}(\Omega)} \leqslant \kappa(\tau)\left\|\overline{\mathrm{y}}_{k-1}-\overline{\mathrm{y}}_{k}\right\|_{L^{2}(\Omega)} \leqslant \varepsilon \kappa(\tau) \sqrt{|\Omega|} \quad(t \in(0, \tau)),
$$

and thus $y(t, x) \geqslant \overline{\mathrm{y}}_{k-1}-\varepsilon \kappa(\tau) \sqrt{|\Omega|}$ on $(0, \tau) \times \Omega$.

Conclusion of the proof. Setting

$$
\varepsilon=\varepsilon(\tau)=\min \left(\frac{\min \left(\overline{\mathrm{y}}_{0}, \mathrm{y}^{1}\right)}{\kappa(\tau) \sqrt{|\Omega|}}, \frac{\overline{\mathrm{y}}_{0}}{1+\kappa(\tau)}\right),
$$

we have obtained a control $u \in L^{2}\left((0, T) \times \Gamma_{0}\right)$, with $T=T_{0}(\varepsilon(\tau))+(K(\varepsilon(\tau))+1) \tau$, such that the solution $y$ of (26) with initial condition $\mathrm{y}^{0}$ and control $u$ satisfies $y(t, x) \geqslant 0$ and $y(T, x)=\mathrm{y}^{1}$.

Remark 15. Assuming moreover that $\inf _{\Omega} \mathrm{y}^{0}>0$, it can be also proved that, for every $\tau>0$, there exists a control $v$ of arbitrary regularity steering the solution (26) from $\mathrm{y}^{0} \geqslant 0$ in $L^{2}(\Omega)$ to $\mathrm{y}^{1} \in(0,+\infty)$ in time $\underline{T}\left(\mathrm{y}^{0}, \mathrm{y}^{1}\right)+\tau$. Let us sketch the proof hereafter. We set $\underline{T}=\underline{T}\left(\mathrm{y}^{0}, \mathrm{y}^{1}\right)$.

1. Taking $\varepsilon \in\left(0, \min \left(\mathrm{y}^{1}, \inf _{\Omega} \mathrm{y}^{0}\right)\right)$, there exists a minimal time $\underline{T}^{\varepsilon}$ such that for every $T>\underline{T}^{\varepsilon}$, there exists a control $v^{\varepsilon} \in L^{1}\left((0, T) \times \Gamma_{0}\right)$ such that the solution $y^{\varepsilon}$ of (26) with control $v^{\varepsilon}$ satisfies $y^{\varepsilon}(T)=\mathrm{y}^{1}$ and $y^{\varepsilon} \geqslant \varepsilon$ on $(0, T) \times \Omega$.

It can be checked that $\underline{T}^{\varepsilon} \geqslant \underline{T}$ and that $\underline{T}^{\varepsilon} \rightarrow \underline{T}$ as $\varepsilon \rightarrow 0$. Hence, taking $\varepsilon>0$ small enough, there exists a control $v^{\varepsilon} \in L^{1}\left((0, \underline{T}+\tau / 3) \times \Gamma_{0}\right)$ such that the corresponding solution $y^{\varepsilon}$ of $(26)$ satisfies $y^{\varepsilon}(\underline{T}+\tau / 3)=\mathrm{y}^{1}$ and $y^{\varepsilon} \geqslant \varepsilon$ on $(0, \underline{T}+\tau / 3) \times \Gamma_{0}$.

2. We regularize the control $v^{\varepsilon}$ of the previous step: we design a control $v_{0} \in C^{\infty}((0, \underline{T}+$ $\left.\tau / 3) \times \Gamma_{0}\right)$ such that all derivatives of $v_{0}$ at time $\underline{T}+\tau / 3$ vanish and such that $v_{0}$ is close to $v^{\varepsilon}$ in $L^{1}\left((0, \underline{T}+\tau / 3) \times \Gamma_{0}\right)$ norm. Let $y_{0}$ be the corresponding solution of (26). If $v_{0}$ is close enough to $v^{\varepsilon}$ in $L^{1}\left((0, \underline{T}+\tau / 3) \Gamma_{0}\right)$ norm, then $y_{0} \geqslant 0$ on $(0, \underline{T}+\tau / 3) \Gamma_{0}$ and $\mathrm{y}_{0}^{1}=y_{0}(\underline{T}+\tau / 3)$ is close to $\mathrm{y}^{1}$ in $H^{-1}(\Omega)$ norm.

3. The conclusion is then similar to the one in Proposition 1:

(a) We take the zero control over $(\underline{T}+\tau / 3, \underline{T}+2 \tau / 3)$, in order to regularize $\mathrm{y}_{0}^{1}$. Then the corresponding solution $\mathrm{y}_{1}^{1}$ at time $\underline{T}+2 \tau / 3$ is close to $\mathrm{y}^{1}$ in any Sobolev norm.

(b) We steer $\mathrm{y}_{1}^{1}$ to $\mathrm{y}^{1}$ with a regular control in time $\tau / 3$ having its derivatives equal to 0 at time 0 . If $\mathrm{y}_{1}^{1}$ is close enough to $\mathrm{y}^{1}$, then we can ensure that the corresponding state trajectory stay nonnegative. Note that $\mathrm{y}_{1}^{1}$ can be taken arbitrarily close to $\mathrm{y}^{1}$ (as a consequence of the regularization of $v^{\varepsilon}$ in Step 2 above).

The more general case $y^{0} \geqslant 0$ is open. It is more difficult, due to the fact that the regularization process in Step 2 does not preserve nonnegativity of the trajectory. A way to establish this result would be to take the zero control over some small time interval $(0, \tau)$ so that, at time $\tau$, we have obtained a state $\tilde{\mathrm{y}}^{0}$ which is positive and close to $\mathrm{y}^{0}$. Then we could proceed as above. But the main difficulty is to prove that $\underline{T}\left(\mathrm{y}^{0}, \mathrm{y}^{1}\right)$ is close to $\underline{T}\left(\tilde{\mathrm{y}}^{0}, \mathrm{y}^{1}\right)$, i.e., that the mapping $\mathrm{y} \in L^{2}(\Omega) \mapsto \underline{T}\left(\mathrm{y}, \mathrm{y}^{1}\right)$ is continuous.

\subsection{Internal control with Neumann boundary conditions}

Let $\Omega$ be a bounded domain of $\mathbb{R}^{d}$ with $C^{2}$ boundary. We consider the internally controlled heat equation with Neumann homogeneous boundary conditions

$$
\begin{aligned}
\partial_{t} y(t, x) & =\Delta y(t, x)+\mathbf{1}_{\omega}(x) u(t, x) & & (t>0, x \in \Omega), \\
\partial_{\nu} y(t, x) & =0 & & (t>0, x \in \partial \Omega), \\
y(0, x) & =\mathrm{y}^{0}(x) & & (x \in \Omega),
\end{aligned}
$$


with $\omega$ an open nonempty subset of $\Omega$, support of the control.

Given $\mathrm{y}^{0} \in L^{2}(\Omega)$ such that $\mathrm{y}^{0} \geqslant 0$, the objective is to steer (29) to a given steady-state $\mathrm{y}^{1}$ (with $\mathrm{y}^{1} \neq \mathrm{y}^{0}$ and $\inf _{\Omega} \mathrm{y}^{1}>0$ ), under the nonnegativity state constraint (3), by means of a control $u=u(t, x)$ localized in $\omega$.

Note that, if $\omega=\Omega$, then we have a trivial solution: the control $u(t, x)=\left(\mathrm{y}^{1}-\mathrm{y}^{0}-(T-\right.$ t) $\left.\Delta \mathrm{y}^{0}-t \Delta \mathrm{y}^{1}\right) / T$ for $(t, x) \in[0, T] \times(0,1)$, with $T>0$, steers the solution $y$ of (29) from $\mathrm{y}^{0}$ to $\mathrm{y}^{1}$ in time $T$ with state $\left((T-t) \mathrm{y}^{0}+t \mathrm{y}^{1}\right) / T$, which of course satisfies the state constraint (3).

This strategy holds when $\mathrm{y}^{0} \in H^{2}(\Omega)$ and $\partial_{\nu} \mathrm{y}^{0}=0$ on $\partial \Omega$. When $\mathrm{y}^{0} \in L^{2}(\Omega)$ only, we can use the regularizing property of the heat equation. More precisely, for any $\tau>0$ :

1. We take the zero control over $(0, \tau)$, so that, then, we have to steer some regular $\mathrm{y}_{0}^{0}$ to $\mathrm{y}^{1}\left(\right.$ with $\left.\mathrm{y}_{0}^{0}=y(\tau)\right)$.

2. Setting $\Omega_{\varepsilon}=\Omega \backslash(\partial \Omega+B(0, \varepsilon))$ for some $\varepsilon>0$, the solution $y_{1}$ of (29) with initial condition $y_{1}(0)=\mathrm{y}_{0}^{0}$, with the control $u=\left.\frac{1}{\tau}\left(\mathrm{y}^{1}-\mathrm{y}_{0}^{0}-(\tau-t) \Delta \mathrm{y}_{0}^{0}-t \Delta \mathrm{y}^{1}\right)\right|_{\Omega_{\varepsilon}}$, satisfies $\left.y_{1}(t)\right|_{\Omega_{\varepsilon}}=(\tau-t) / \tau \mathrm{y}_{0}^{0}+t / \tau \mathrm{y}^{1}$, and in $\Omega \backslash \Omega_{\varepsilon}$ it is solution of

$$
\begin{aligned}
\partial_{t} y_{1}(t, x) & =\Delta y_{1}(t, x)+\mathbf{1}_{\omega}(x) u(t, x) & & \left(t>0, x \in \Omega \backslash \Omega_{\varepsilon}\right), \\
\partial_{\nu} y_{1}(t, x) & =0 & & (t>0, x \in \partial \Omega), \\
y_{1}(t, x) & =\frac{\tau-t}{\tau} \mathrm{y}_{0}^{0}+\frac{t}{\tau} \mathrm{y}^{1} & & \left(t>0, x \in \partial \Omega_{\varepsilon}\right), \\
y_{1}(0, x) & =\mathrm{y}_{0}^{0}(x) & & \left(x \in \Omega \backslash \Omega_{\varepsilon}\right) .
\end{aligned}
$$

Now, setting $\mathrm{y}_{1}^{0}=y_{1}(\tau)$, we have $\left\|\mathrm{y}_{1}^{0}-\mathrm{y}^{1}\right\|_{L^{2}(\Omega)} \leqslant \sqrt{\left|\Omega \backslash \Omega_{\varepsilon}\right|}\left\|\mathrm{y}_{0}^{0}-\mathrm{y}^{1}\right\|_{L^{\infty}(\Omega)}$, which converges to 0 as $\varepsilon \rightarrow 0$.

3. For $\varepsilon>0$ small enough, one can steer $\mathrm{y}_{1}^{0}$ to $\mathrm{y}^{1}$ in time $\tau$ while preserving nonnegativity of the trajectory. This result is a consequence of Lemma 3 (adapted to internal control).

We now assume that $\omega \neq \Omega$. Then we have the following result, similarly as before: there exist $T>0$ and a control $u \in L^{2}((0, T) \times \omega)$ such that the solution $y$ of $(29)$, with $y(0)=\mathrm{y}^{0}$, satisfies $y(T)=\mathrm{y}^{1}$ and the nonnegativity state constraint (3) (same argument as in the proof of Theorem 5 in Section 4.2). Then, defining as before the minimal time, we have $\underline{T}\left(\mathrm{y}^{0}, \mathrm{y}^{1}\right)>0$. Indeed, restricting the solution of (29) to a ball contained in $\Omega \backslash \omega$ and taking the trace on the boundary of this ball, leads to a Dirichlet problem as the one studied in Section 3.1 and it has been shown in Theorem 3 that this control problem with nonegative control constraint cannot be solved within arbitrarily small time.

Consequently, we have proved that, if $\left.\mathrm{y}^{0}\right|_{\Omega \backslash \omega} \neq\left.\mathrm{y}^{1}\right|_{\Omega \backslash \omega}$, then $\underline{T}\left(\mathrm{y}^{0}, \mathrm{y}^{1}\right)>0$.

Remark 16. If $\left.\mathrm{y}^{0}\right|_{\Omega \backslash \omega}=\left.\mathrm{y}^{1}\right|_{\Omega \backslash \omega}$ and $\left.\mathrm{y}^{0}\right|_{\omega} \neq\left.\mathrm{y}^{1}\right|_{\omega}$, then $\underline{T}\left(\mathrm{y}^{0}, \mathrm{y}^{1}\right)=0$. In fact, if $\mathrm{y}^{0} \in H^{2}(\Omega)$, $\left.\mathrm{y}^{0}\right|_{\Omega \backslash \omega}=\left.\mathrm{y}^{1}\right|_{\Omega \backslash \omega}$ and $\partial_{\nu} \mathrm{y}^{0}=0$ on $\partial \Omega$, then for any $T>0$, the control $u=\left(\mathrm{y}^{1}-\mathrm{y}^{0}-(T-t) \Delta \mathrm{y}^{0}-\right.$ $\left.t \Delta \mathrm{y}^{1}\right) / T$ is supported in $\omega$ and steers $\mathrm{y}^{0}$ to $\mathrm{y}^{1}$ in time $T$ (and the associated trajectory $\left((T-t) \mathrm{y}^{0}+t \mathrm{y}^{1}\right) / T$ is nonnegative $)$.

The general case $\mathrm{y}^{0} \in L^{2}(\Omega)$ can be treated with the same strategy as the one used to prove that $\underline{T}\left(\mathrm{y}^{0}, \mathrm{y}^{1}\right)=0$ when $\omega=\Omega$.

Remark 17. We have presented here the result with Neumann homogeneous boundary condition. But the boundary conditions do not affect the result on the positivity of $\underline{T}\left(\mathrm{y}^{0}, \mathrm{y}^{1}\right)$.

\subsection{Neumann boundary control with control constraints in 1D}

Let $\mathrm{y}^{0} \in \mathbb{R}$ and $\mathrm{y}^{1} \in \mathbb{R}$ be two real numbers with $\mathrm{y}^{0} \neq \mathrm{y}^{1}$. We consider the $1 \mathrm{D}$ heat equation with Neumann boundary controls:

$$
\begin{array}{llrl}
\partial_{t} y(t, x) & =\partial_{x}^{2} y(t, x) & & (t>0, x \in(0,1)), \\
\partial_{x} y(t, 0) & =v_{0}(t) & & (t>0), \\
\partial_{x} y(t, 1) & =v_{1}(t) & & (t>0),
\end{array}
$$


with constant initial condition $y(0, x)=\mathrm{y}^{0}$ (steady-state). We consider the question of knowing if it is possible or not to steer (1) to $\mathrm{y}^{1}$ with under the nonnegativity control constraints

$$
v_{0}(t) \geqslant 0 \quad \text { and } \quad v_{1}(t) \geqslant 0 \quad(t>0 \text { a.e. }) .
$$

The following result shows that this is impossible.

Theorem 6. Given any $T>0$, there do not exist any controls $v_{0}$ and $v_{1}$ in $\mathcal{M}(0, T)$ that are nonnegative Radon measure, such that the corresponding solution $y$ of (25) satisfies $y(0)=\mathrm{y}^{0}$ and $y(T)=\mathrm{y}^{1}$.

In Section 4.2, we considered Neumann boundary controls with constraints on the state. The results of the present section are of complementary interest since they show that different constraints can have various effects on the control property, making it impossible to achieve in the Neumann case with constraints on the control.

Proof. We follow the arguments of Theorem 2. Assume by contradiction that there exist $T>0$ and controls $v_{0}$ and $v_{1} \in \mathcal{M}(0, T)$ satisfying (31) such that the solution $y$ of (25) satisfies $y(0)=\mathrm{y}^{0}$ and $y(T)=\mathrm{y}^{1}$. Defining $y_{n}(t)=\int_{0}^{1} y(t, x) \cos (n \pi x) \mathrm{d} x$ for $t \in[0, T]$ and $n \in \mathbb{N}$, we have, by integrations by parts, $\dot{y}_{n}(t)=-n^{2} \pi^{2} y_{n}(t)+(-1)^{n} v_{1}(t)-v_{0}(t)$, and thus

$$
y_{n}(T)=e^{-n^{2} \pi^{2} T} y_{n}(0)+\int_{0}^{T} e^{-n^{2} \pi^{2}(T-t)} \mathrm{d}\left((-1)^{n} v_{1}-v_{0}\right)(t)
$$

Since $y(0)=\mathrm{y}^{0}$ and $y(T)=\mathrm{y}^{1}$, we must have $y_{0}(0)=\mathrm{y}^{0}$ and $y_{n}(0)=0$ if $n>0$, and $y_{0}(T)=\mathrm{y}^{1}$ and $y_{n}(T)=0$ if $n>0$. Hence,

$$
\begin{aligned}
\mathrm{y}^{1}-\mathrm{y}^{0} & =\int_{[0, T]} \mathrm{d}\left(v_{1}-v_{0}\right)(t), & \\
0 & =\int_{[0, T]} e^{-(2 p)^{2} \pi^{2}(T-t)} \mathrm{d}\left(v_{1}-v_{0}\right)(t) & \left(p \in \mathbb{N}^{*}\right) \\
0 & =\int_{[0, T]} e^{-(2 p+1)^{2} \pi^{2}(T-t)} \mathrm{d}\left(v_{1}+v_{0}\right)(t) & \left(p \in \mathbb{N}^{*}\right) .
\end{aligned}
$$

The conditions (32c) and (31) lead to $v_{0}=v_{1}=0$, which is incompatible with the first condition (32a) $\left(\right.$ since $\left.\mathrm{y}^{0} \neq \mathrm{y}^{1}\right)$. We get a contradiction.

Remark 18. We have stated the negative controllability result with controls in $\mathcal{M}(0, T)$ which is the most general class to consider nonnegative controls. As we see in the proof, the Fourier series expansion is well justified in that control setting since the duality between controls and the time real exponentials (which are continuous) is well justified.

Remark 19. The result of Theorem 6 is valid as well when both controls are of constant sign with respect to $t$ but not necessarily the same.

Some remarks are in order on the negative result of Theorem 6.

Remark 20. An alternative argument of proof is the following. If there were to exist nonnegative nontrivial controls $v_{0}$ and $v_{1}$, then, for $p \in \mathbb{N}$, the $(2 p+1)^{\text {th }}$ Fourier coefficient of $y(T, \cdot)$, namely $\int_{0}^{1} y(T, x) \cos ((2 p+1) \pi x) \mathrm{d} x$, would be positive. In other words, the action of the controls generates some Fourier modes which cannot be cancelled. Since the modes associated with the final target $y(T)=\mathrm{y}^{1}$ are zero, this would lead to a contradiction.

Remark 21. Still, one can wonder why, as it occurs in the context of Dirichlet control, the constrained controllability property cannot be guaranteed if the control time is long enough.

To prove controllability under constraints in long time, one could use a quasi-static strategy (see [6] and [5, Chapter 7]), which consists first on considering a path of equilibria joining the initial and final data. The path of equilibria in this case is $\bar{y}(\tau)=(1-\tau) \mathrm{y}^{0}+\tau \mathrm{y}^{1}$, the corresponding Neumann traces are the trivial ones $\bar{v}_{0}(\tau)=\bar{v}_{1}(\tau)=0$, for $\tau \in[0,1]$, and they lie on the boundary of the constraints that we impose. In other words, the path of pairs 
state-controls $\tau \in[0,1] \mapsto\left(\overline{\mathrm{y}}(\tau), \bar{v}_{0}(\tau), \bar{v}_{1}(\tau)\right) \in L^{2}(0,1) \times \mathbb{R}^{2}$ does not belong to the interior of the set $L^{2}(0,1) \times(0,+\infty)^{2}$. This causes the failure of the quasi-static control strategy, which requires adding extra controls to correct the defect of the trajectory when satisfying the heat equation.

Remark 22. The argument based on using a translation of the state and the fact that the observability constant decays as $T$ tends to infinity fails as well. Indeed, let us introduce the new state $z=y-\mathrm{y}^{1}$. The goal is to drive $z$ from $\mathrm{y}^{0}-\mathrm{y}^{1}$ to zero in time $T$. This can be achieved by means of boundary controls $w_{0}(t)$ and $w_{1}(t)$, and they can be shown to be small in $L^{\infty}(0, T)$ if the control time $T$ is large enough. But these controls are of oscillatory nature and they cannot be guaranteed to keep a constant sign. Going back to the original state $y$ and due to the boundary conditions of Neumann type, we observe that the Neumann controls for $y$ are precisely $w_{0}(t)$ and $w_{1}(t)$. This translation argument does not allow to conclude the Neumann control with nonnegative controls even if $T$ is large. But, in fact, as shown above, this is impossible whatever $T$ is.

Remark 23. Whether the negative $1 \mathrm{D}$ result of Theorem 6 is valid also in multi-D case is an open question.

\section{$5 \quad$ Numerical simulations}

In this section we run some numerical simulations to illustrate our results. We focus on the 1D heat equation with Neumann or Dirichlet boundary controls. To simplify the presentation, we will take positive and constant initial and final states $\mathrm{y}^{0}$ and $\mathrm{y}^{1}$.

According to Remark 11 and as a consequence of the comparison principle for the solutions of the heat equation, the minimal time for the control of the heat equation with state constraints coincides with the minimal time arising when the constraints are imposed only on the control. According to Lemma 2 in Section 2.6, in both situations, this minimal time satisfies lower estimates given by (10). Moreover, for the Dirichlet control problem we can take $u_{0}=u_{1}$, and for the Neumann control problem we can take $v_{0}=-v_{1}$.

\subsection{Dirichlet boundary controls with nonnegativity control con- straints}

Let us consider the minimal time control problem for the $1 \mathrm{D}$ heat equation on $(0,1)$ with Dirichlet boundary controls submitted to a nonnegativity constraint. In order to perform numerical simulations, we choose the simplest possible discretization scheme: finite differences in space, Euler explicit scheme in time, with a uniform space-time grid $t_{i}=i \frac{T}{N_{t}}, i=0, \ldots, N_{t}$, $x_{j}=\frac{i}{N_{x}}, j=0, \ldots, N_{x}$, where $N_{t}$ and $N_{x}$ are positive integers satisfying the Courant-FriedrichLewy condition $2 \Delta t \leqslant(\Delta x)^{2}$, with $\Delta t=T / N_{t}$ and $\Delta x=1 / N_{x}$.

In all the numerical examples presented hereafter, we will have $T<0.25$, and we choose $N_{x}=30$ and $N_{t}=450$.

The discrete state is then a $\left(N_{t}+1\right) \times\left(N_{x}+1\right)$-component discrete matrix $\left(Y_{i, j}\right)$ representing the approximation of $y(t, x)$ over the grid-points, the two $\left(N_{t}+1\right)$-component column vectors $\left(U_{i}\right)$ and $\left(V_{i}\right)$ representing the discretized controls both at $x=0$ and $x=1$, and the scalar $T \geqslant 0$ which is the final time. These discrete states-controls are linked by the discrete relations representing the dynamics, the boundary constraints and terminal condition at $t=T$. The resulting optimization problem, under discrete control-constraints, is the following:

minimize $T$ 
under the constraints

$$
\begin{array}{ll}
\frac{Y_{i+1, j}-Y_{i, j}}{\Delta t}=\frac{Y_{i, j+1}-2 Y_{i, j}+Y_{i, j-1}}{(\Delta x)^{2}}, & j=1, \ldots, N_{x}-1, i=0, \ldots, N_{t}-1, \\
Y_{i, 0}=U_{i}^{0}, Y_{i, N_{x}}=U_{i}^{1}, & i=0, \ldots, N_{t}, \\
U_{i}^{0} \geqslant 0, U_{i}^{1} \geqslant 0, & i=0, \ldots, N_{t}, \\
Y_{0, j}=\mathrm{y}^{0}, Y_{N_{t}, j}=\mathrm{y}^{1}, & j=1, \ldots, N_{x}-1 .
\end{array}
$$

This is a standard finite-dimensional constrained optimization problem, in dimension that is larger as the discretization is finer. To solve it numerically, we use the expert interiorpoint optimization routine Ip0pt (see [26]) combined with automatic differentiation and the modelling language AMPL (see [9]). We refer to [1, 20, 21] for a survey on numerical methods in optimal control and how to implement them efficiently according to the context.

The interest of the simulations we perform hereafter is justified by the convergence result in Section 5.3 (see further) that guarantees that the discrete optimal time converges, as the mesh-size tends to zero, to the minimal time for the continuous constrained control problem.

Case $\mathrm{y}^{0} \equiv 5$ and $\mathrm{y}^{1} \equiv 1$. According to Section 2 and Example 1, we must have $T \geqslant$ 0.165297 . Figure 2 displays the numerical solution of the above problem, with $N_{t}=450$ and $N_{x}=30$. 

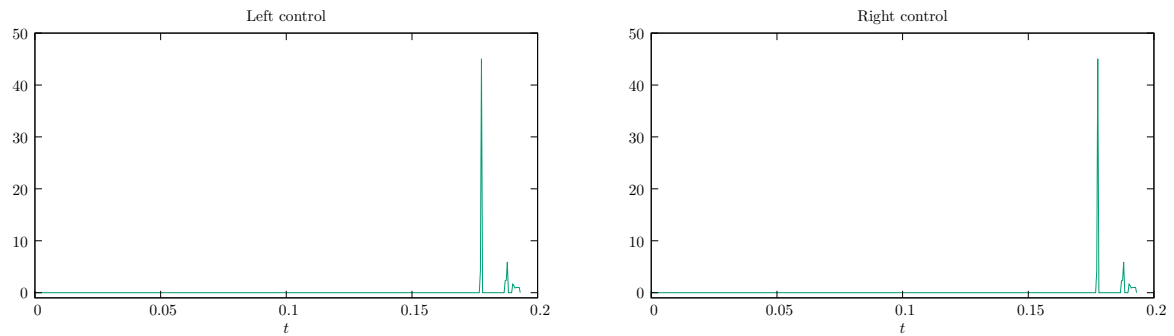

(a) Time evolution of the boundary controls.

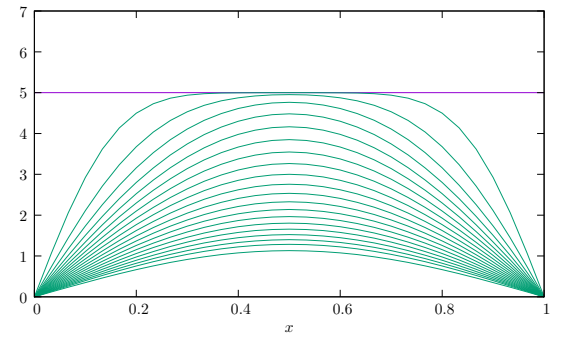

(b) Evolution of the state between times 0 and 0.1764 .

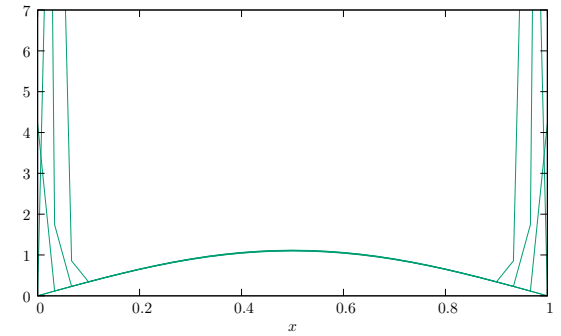

(c) Evolution of the state between times 0.1764 and 0.1781 .

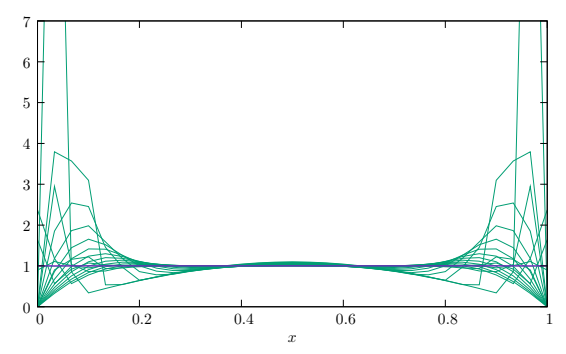

(d) Evolution of the state between times 0.1781 and the final time, 0.1931 .

Figure 2: Evolution of the controls and of the state from the initial value $(=5)$ to the final one (=1) in the minimal computed time $T \simeq 0.1931$.

The minimal time we obtain from our simulations is $T \simeq 0.1931$, which is compatible with the theoretical lower bound prediction, but larger, which is consistent with the fact that our analysis yields only lower bounds that, probably, are not sharp. In particular, we observe that, taking larger $N_{t}$ and $N_{x}$, the value of the resulting minimal time does not change significantly. This is in accordance with the convergence result given in $\S 5.3$ of the minimal time for the discretized problem, to the minimal time of the continuous one, as $N_{t}$ and $N_{x}$ tend to $+\infty$ (preserving the CFL condition).

It is interesting to note on Figure 2 that the two controls coincide and are identically equal to 0 over long time subintervals. We have shown that the controls can always be chosen to coincide; but, in general, they are not unique. And yet, the controls we obtain in our numerical experiments are always symmetric. This raises the question of knowing whether, at the minimal time, the controls are unique (if so, they must be equal). The numerical simulations also raise the interesting question of whether the controls in the minimal control time necessarily present a "sparse" structure with long lags where they are identically zero.

The evolution of the state that we observe on Figure 2 presents the following features:

- The solution starts from the constant initial state $\mathrm{y}^{0} \equiv 5$. 
- Since we first have $u_{0}(t)=u_{1}(t)=0$ during a significantly long time subinterval, the solution follows the dynamics of the heat equation with null boundary conditions, in the absence of controls (see Figure 2(b)).

Using the Fourier expansion of the solutions, the state trajectory on this time interval can be computed and is given by

$$
y(t, x)=4 \mathrm{y}^{0} \sum_{p=0}^{\infty} \frac{e^{-(2 p+1)^{2} \pi^{2} t}}{(2 p+1) \pi} \sin ((2 p+1) \pi x) .
$$

we observe that, during this time interval, the state approximately coincides with the first Fourier mode, i.e., $y(t, x) \simeq \frac{20}{\pi} e^{-\pi^{2} t} \sin (\pi x)$. In agreement with this analytical observation the state drawn in blue looks as a concave function, vanishing at the boundaries $x=0$ and $x=1$. The maximum of this function, which is symmetric with respect to at $x=1 / 2$, is reached precisely at $x=1 / 2$, and this maximum decreases as time increases.

- These dynamics, the one of the free heat equation with homogeneous Dirichlet boundary conditions, remain so until $\max _{x} y\left(t_{1}, x\right)=y\left(t_{1}, 1 / 2\right) \simeq 1$, that is, at $t_{1} \simeq 0.1764$. Then, the control takes large positive values (over a short time interval $(0.1764,0.1781)$ ), and, accordingly, so does the solution at the boundary points $x=0$ and $x=1$ (see Figure $2(\mathrm{c})$ ). After this short interval, the solution adjusts itself to reach exactly $\mathrm{y}^{1}=1$ (see Figure 2(d)).

- Controls present an "off-bang-off-bang-off" structure. In other words, roughly, the optimal (minimal time) strategy consists of, first, letting the solution damp itself out, exponentially decrease to 0 while staying positive; when the solution becomes small enough (just below 1 but touching 1 at its maximum), the control switches on taking positive values to increase the solution, on the left and on the right, and make it match the desired target solution.

This strategy corresponds to intuition, and follows from the nonnegativity control constraints. Other numerical simulations, not reported here, confirm that, as expected, if we remove this nonnegativity control constraint, then one can steer $\mathrm{y}^{0}=5$ to $\mathrm{y}^{1}=1$ in arbitrarily small time with oscillating (changing sign) controls.

Case $\mathrm{y}^{0} \equiv 1$ and $\mathrm{y}^{1} \equiv 5$. According to Section 2 and Example 1 , we must have $T>$ 0.023076. We adopt the same discretization scheme, with $N_{x}=20$. Moreover, we add temporarily an upper constraint on the controls, assuming that $0 \leqslant u_{0}(t) \leqslant M$ and $0 \leqslant u_{1}(t) \leqslant M$ for some $M>0$ that, in practice, is chosen large enough. We do so because our theoretical results predict that controls may be Radon measures including some singular components (Dirac deltas). If that were the case, the possible need of Dirac masses should be visible in the numerical experiments by taking $M$ to be larger and larger. This is precisely what is observed in our numerical simulations (see Figures 3 and 4).

Consider first Figure 3. The minimal time we obtain is $T \simeq 0.0498$, which is compatible with the theoretical prediction $(T>0.02307)$. In this numerical simulation, the optimal strategy develops the following features:

- We start with a bang arc along which $u_{0}(t)=u_{1}(t)=50=M$, the maximal authorized value (see the corresponding state trajectory on Figure 3(b)), which is compatible with the possible presence of Dirac components on the controls at the initial time.

Along this arc, at any time instance, the solution looks as a convex function, equal to 50 at both boundaries $x=0$ and $x=1$.

Using Fourier expansion, the state trajectory on this time interval can be computed and at the final time of this arc, $t_{1} \simeq 0.0115$, the state is given by

$$
y\left(t_{1}, x\right)=M+4\left(\mathrm{y}^{0}-M\right) \sum_{p=0}^{\infty} \frac{e^{-(2 p+1)^{2} \pi^{2} t_{1}}}{(2 p+1) \pi} \sin ((2 p+1) \pi x) .
$$



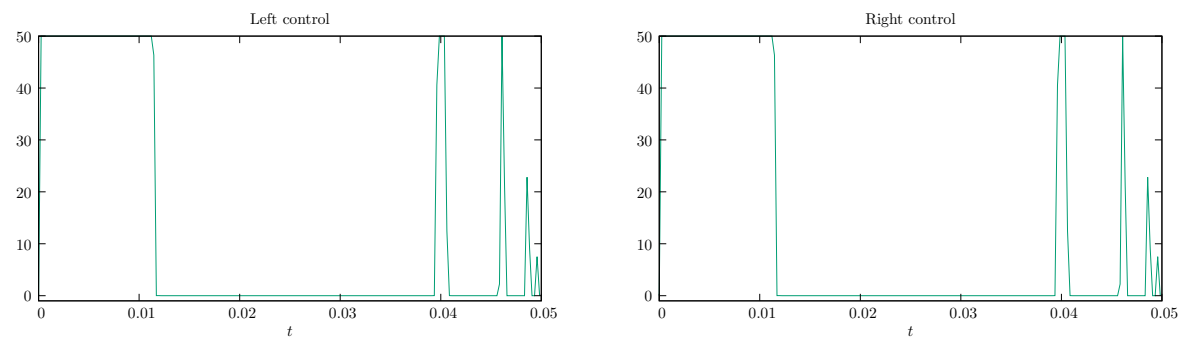

(a) Time evolution of the boundary controls.

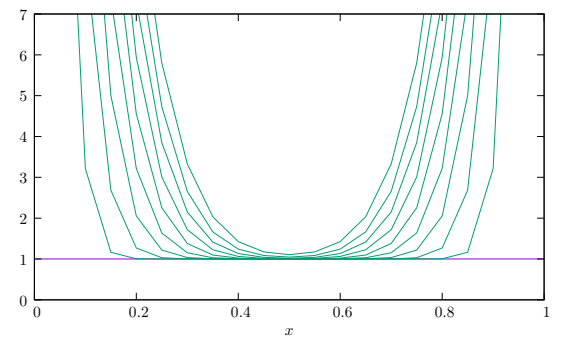

(b) Evolution of the state between times 0 and 0.0115 .

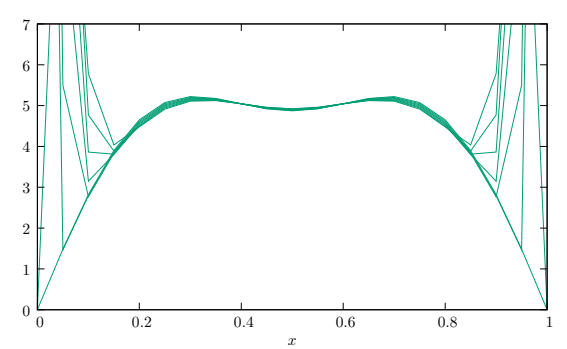

(d) Evolution of the state between times 0.0394 and 0.0409 .

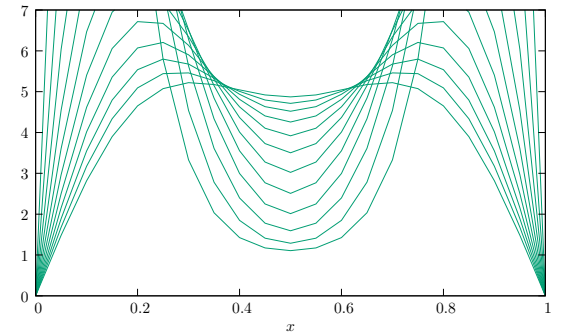

(c) Evolution of the state between times 0.0115 and 0.0394 .

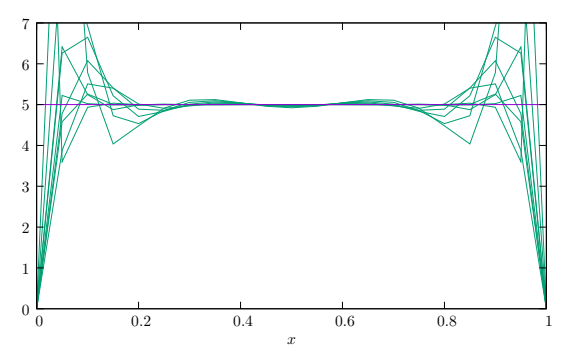

(e) Evolution of the state between times 0.0409 and the final time, 0.0498 .

Figure 3: $M=50$ : Evolution of the controls and of the state from the initial value $(=5)$ to the final one $(=1)$ in the minimal computed time $T \simeq 0.0498$.

- Then the controls switch to $u_{0}(t)=u_{1}(t)=0$ over a quite long subinterval (compared to the total length of the control horizon $[0, T]$ ) and then the solution vanishes at the two boundaries, looking as an inverted double potential (see the corresponding state trajectory on Figure 3(c)). During this time interval, the solution can also be computed, using Fourier expansion and is given by

$$
\begin{aligned}
y(t, x)=4 M \sum_{p=0}^{\infty} \frac{e^{-(2 p+1)^{2} \pi^{2}\left(t-t_{1}\right)}}{(2 p+1) \pi} \sin ((2 p & +1) \pi x) \\
& +4\left(\mathrm{y}^{0}-M\right) \sum_{p=0}^{\infty} \frac{e^{-(2 p+1)^{2} \pi^{2} t}}{(2 p+1) \pi} \sin ((2 p+1) \pi x) .
\end{aligned}
$$



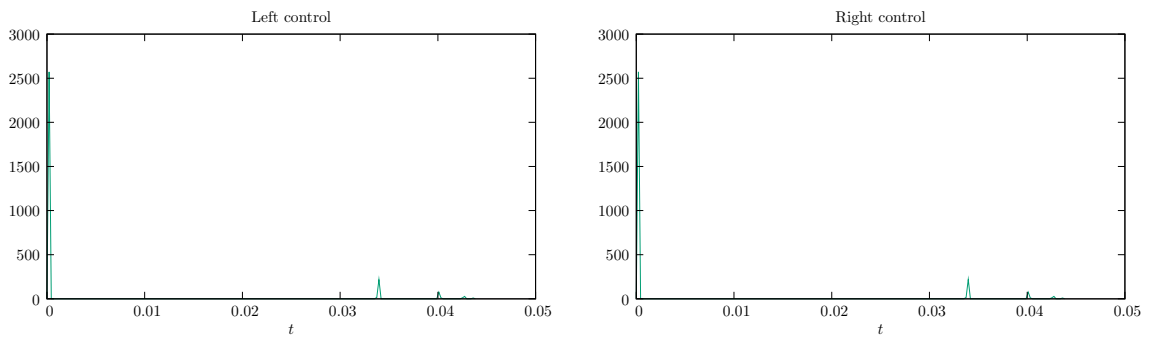

(a) Time evolution of the boundary controls.

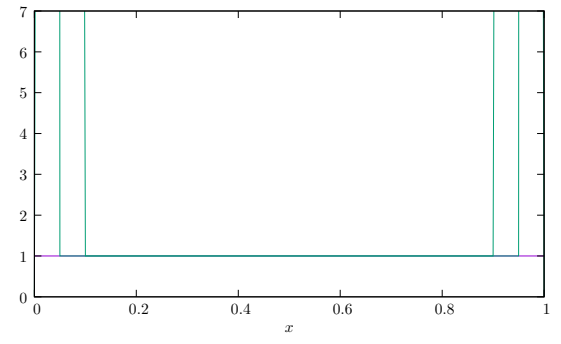

(b) Evolution of the state between times 0 and 0.0004 .

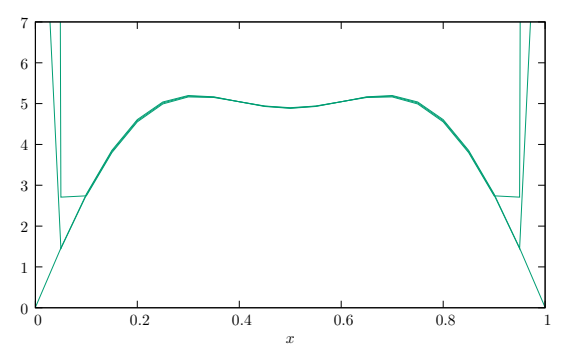

(d) Evolution of the state between times 0.0335 and 0.0340 .

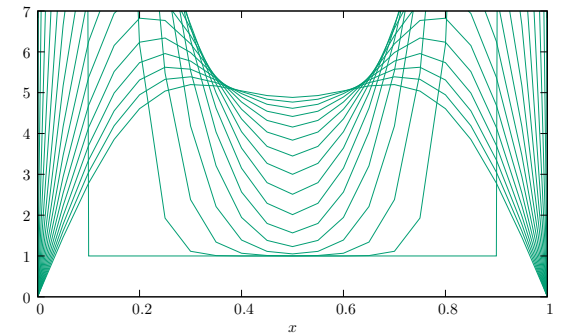

(c) Evolution of the state between times 0.0004 and 0.0335 .

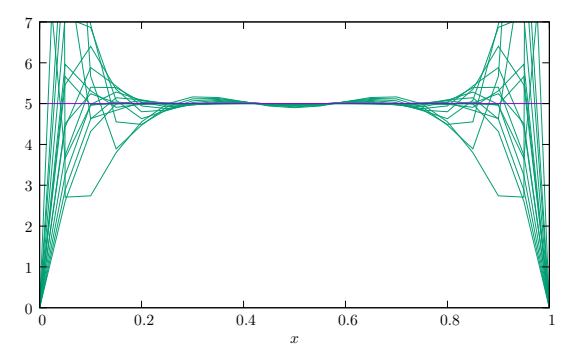

(e) Evolution of the state between times 0.0340 and the final time, 0.0438 .

Figure 4: $M=+\infty$ : Evolution of the controls and of the state from the initial value $(=5)$ to the final one $(=1)$ in the minimal computed time $T \simeq 0.0438$.

Approximating this solution with the first three Fourier modes,

$$
\begin{aligned}
& y(t, x) \simeq\left(M+\left(\mathrm{y}^{0}-M\right) e^{-\pi^{2} t_{1}}\right) \frac{4 e^{-\pi^{2}\left(t-t_{1}\right)} \sin (\pi x)}{\pi} \\
&+\left(M+\left(\mathrm{y}^{0}-M\right) e^{-9 \pi^{2} t_{1}}\right) \frac{4 e^{-9 \pi^{2}\left(t-t_{1}\right)} \sin (3 \pi x)}{3 \pi},
\end{aligned}
$$

we recover the inverted double potential shape of the solution.

- At a later time $t_{1}$, that can be easily observed in the discrete dynamics, the solution becomes concave, less than 5 and touching 5 at $x=1 / 2$. Then the control switch and take positive values again, to adjust the solution to the final target $\mathrm{y}^{1}=5$ (see Figures $3(\mathrm{~d}$ ) and $3(\mathrm{e}))$.

Consider now Figure 4. It corresponds to the limit situation $M=+\infty$. The previous first bang arc $u_{0}=u_{1}=50$ now becomes a very short arc along which the controls take a very large value, which is compatible with an approximation of a Dirac impulse. This is in accordance 
with our theoretical result, predicting that controls could belong to the set of Radon measures, and develop some singularities.

It is likely that the seemingly short impulse that one can see for the controls at $t \simeq 0.037$ is actually a Dirac mass, but the discretization is not fine enough to reproduce this fact faithfully.

Turnpike and/or sparsity structure? The structure of the controls observed in these simulations reveal a turnpike and sparse structure, meaning that the optimal trajectory, defined on $[0, T]$ approximately consists of three parts:

- A first short-time arc, on $[0, \varepsilon]$ with $\varepsilon \ll T$, along which the control possibly takes maximal values,

- A middle arc, on $[\varepsilon, T-\varepsilon]$, where the control remains in a steady configuration (the null one),

- A final short-time arc, on $[T-\varepsilon, T]$, along which the control possibly takes maximal values.

The turnpike phenomenon was analyzed in [23] for general nonlinear optimal control problems in finite dimension, showing that, when one steers a control system from one initial configuration to a final configuration in fixed time $T$, then, for $T$ large, the optimal trajectory enjoys the above qualitative behavior, and more precisely, the long middle arc corresponds to an optimal steady-state, i.e., the optimal solution of an associated static optimal control problem. We have even proved that, except at the beginning and at the end of the interval, if $T$ is large enough, the solution, the control, and the adjoint vector (coming from the application of the Pontryagin maximum principle) are exponentially close to some constant values, corresponding to the solution of the static version of the optimal control problem. We have recently extended this analysis in [22] to infinite dimension, involving in particular the case of heat equations (see also [10] for a specific analysis on the wave equation).

Here, however, the context is slightly different because we minimize the time, and therefore the final time $T$ is not expected to be large as in the above-mentioned references. Still, optimal solutions and control seem to develop a similar behavior.

The optimal controls present long lags where they take null values, saturating the constraint. Thus, their structure is of sparse nature, which is due to the fact that the control time is minimized. In recent years the existence of sparse controls has been derived for a variety of parabolic control problems but, normally, under the constraint that they are of minimal norm in the space of measures, a fact that enhances their concentration into Dirac deltas (see [4]). Here however, the sparsity seems to be due to the fact that controls are constrained by the nonnegativity condition and the time is minimal. This issue requires further analysis and understanding.

\subsection{Neumann controls and state constraints}

Let us now consider the minimal time control problem for the $1 \mathrm{D}$ heat equation on $(0,1)$ with Neumann boundary controls, under a nonnegativity constraint on the state $y(t, x) \geqslant 0$. Note that in this case we expect the states to be those that we obtain by imposing nonnegativity constraints on the Dirichlet controls. Accordingly, the Neumann controls in the minimal time are expected to be the normal traces of the Dirichlet controlled trajectories. Our numerical experiments hereafter confirm this fact.

We choose $\mathrm{y}^{0} \equiv 5$ and $\mathrm{y}^{1} \equiv 1$ and the following discretization:

$$
\begin{array}{ll}
\frac{Y_{i+1, j}-Y_{i, j}}{\Delta t}=\frac{Y_{i, j+1}-2 Y_{i, j}+Y_{i, j-1}}{(\Delta x)^{2}}, & j=1, \ldots, N_{x}-1, i=0, \ldots, N_{t}-1, \\
\frac{Y_{i, 1}-Y_{i, 0}}{\Delta x}=V_{i}^{0}, \frac{Y_{i, N_{x}}-Y_{i, N_{x}-1}}{\Delta x}=V_{i}^{1}, & i=0, \ldots, N_{t}, \\
Y_{i, j} \geqslant 0, j=0, \ldots, N_{x}, & i=0, \ldots, N_{t}, \\
Y_{0, j}=5, Y_{N_{t}, j}=1, & j=0, \ldots, N_{x} .
\end{array}
$$


As before we add an upper bound on the controls, $\left|v_{0}(t)\right| \leqslant M$ and $\left|v_{1}(t)\right| \leqslant M$, with $M>0$ to be chosen large.

On Figure 5, we report the numerical results obtained for $M=20$. The minimal time that is obtained is then $T \simeq 0.2087$.
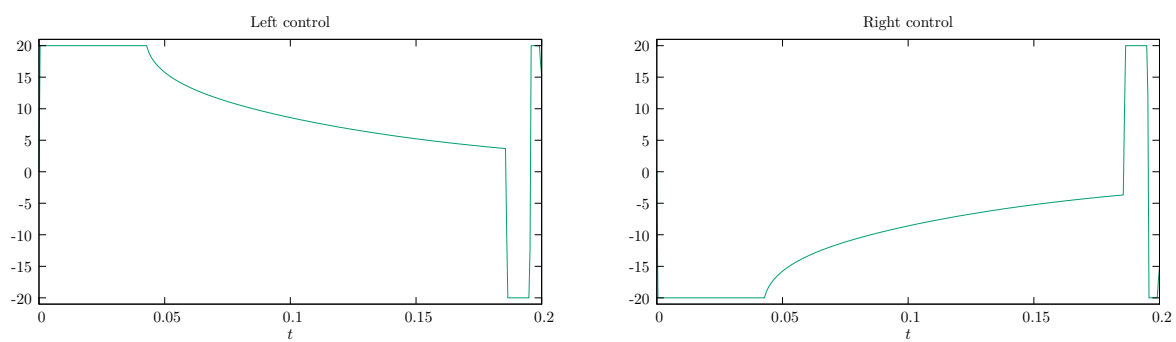

(a) Time evolution of the boundary controls.

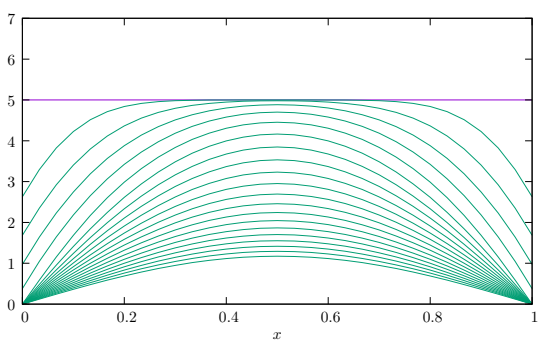

(b) Evolution of the state between times 0 and 0.1856 .

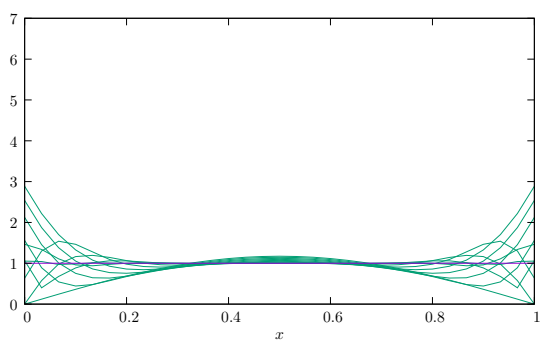

(c) Evolution of the state between times 0.1856 and the final time, 0.2093 .

Figure 5: Evolution of the controls and of the state from the initial value $(=5)$ to the final one $(=1)$ in the minimal computed time $T \simeq 0.2093$.

The optimal strategy starts with a bang arc with $v_{0}(t)=20$ and $v_{1}(t)=-20$. Once more, we observe the odd symmetry $v_{0}=-v_{1}$ of the controls (see Figure 5(a)). Along this arc, starting from $\mathrm{y}^{0} \equiv 5$, the solution is concave, symmetric with respect to $x=1 / 2$, and takes boundary values at $x=0$ and $x=1$, that are positive and decrease in time (see Figure $5(\mathrm{~b})$ ).

When these values reach 0 , the controls switch and then evolve continuously, not saturating the constraints. This arc is therefore singular, which is a very interesting feature. Along this singular arc, the solution remains concave, vanishes at both boundaries $x=0$ and $x=1$, and its maximum decreases (see Figure Figure 5(b)). This is so, until its maximum reaches the target value 1 .

Then the controls switch again, and oscillate much (they switch between +20 and -20 ) before the solution finally reaches the target $\mathrm{y}^{1} \equiv 1$ (see Figure $5(\mathrm{c})$ ). A chattering phenomenon seems to occur just before the final time, and the controls switch very rapidly over a compact time interval. This could be a manifestation of the fact that, for the continuous problem controls switch infinitely many times.

On Figure 6, we report what happens for $M=+\infty$. The minimal time that is obtained is $T \simeq 0.1938$. This situation corresponds to the limit of the previous one as $M \rightarrow+\infty$. We observe that the first arc converges to a Dirac mass, and oscillations between -20 and 20 at the end of the interval are replaced by oscillations of negative and positive Dirac impulses.

We observe that the minimal time obtained and the state trajectory plot on Figure 6 are in accordance with the one obtained for the Dirichlet control case (see Figure 2). As explained in Section 4.2, this fact was expected. 

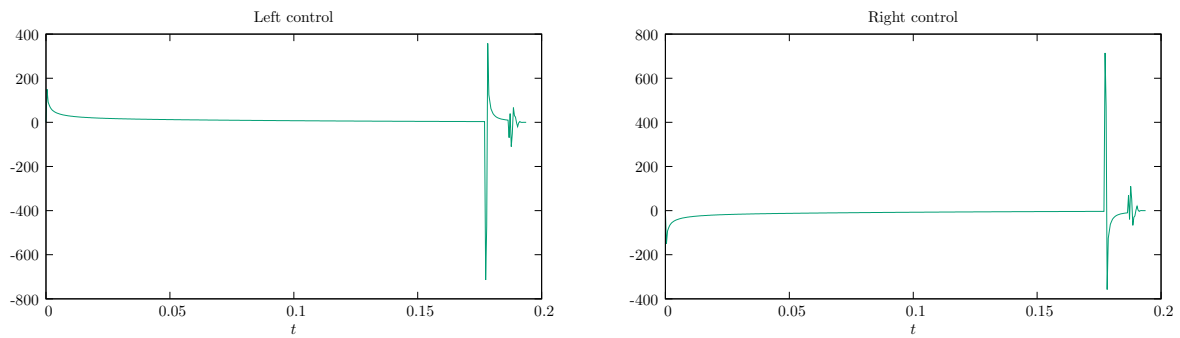

(a) Time evolution of the boundary controls.

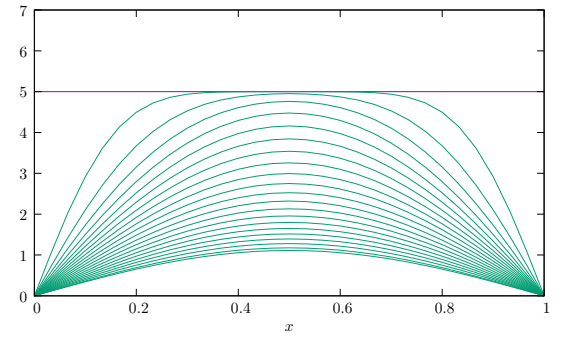

(b) Evolution of the state between times 0 and 0.1764 .

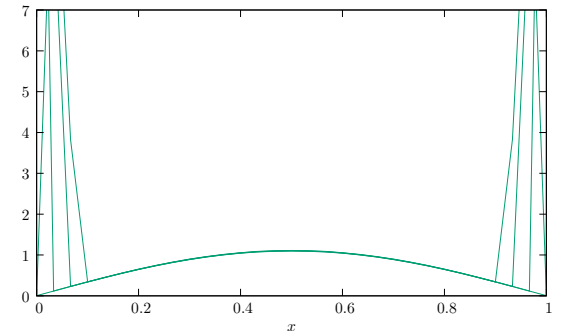

(c) Evolution of the state between times 0.1770 and 0.1783 .

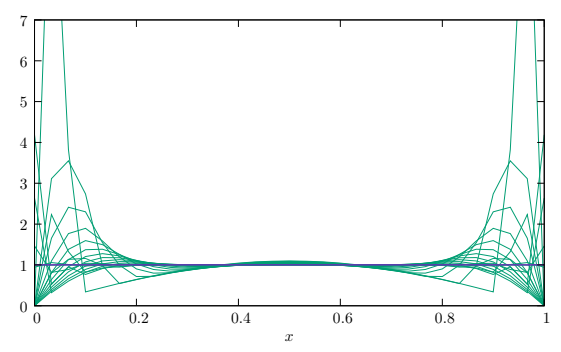

(d) Evolution of the state between times 0.1783 and the final time, 0.1938 .

Figure 6: Evolution of the controls and of the state from the initial value $(=5)$ to the final one (=1) in the minimal computed time $T \simeq 0.1938$.

We can still observe the middle "singular" arc. As before, this is also a kind of turnpike and sparsity phenomenon, but this time, of a different nature, because the long middle arc is a singular arc.

\subsection{Convergence result for the minimal time}

The numerical simulations that we have developed here exhibit the expected phenomena for the constrained control problems under consideration. The validity of these computational results can be confirmed by a convergence result showing that the minimal time of control for the discrete problem converges towards the discrete one as the mesh-sizes tend to zero.

Here we briefly sketch the main ideas of a possible proof. Completing the details would require further analytical work combining the ingredients developed in the existing literature.

We denote by $T$ the minimal time of control under constrains for fixed $\mathrm{y}^{0}$ and $\mathrm{y}^{1}$, as above, for the continuous heat equation. We denote similarly by $T_{N}$ the minimal time of control for the discrete problem corresponding to the mesh-size parameters $N_{x}$ and $N_{t}$ satisfying the CFL 
condition for stability. We are interested in the convergence of $T_{N}$ towards $T$, as $N_{x}$ and $N_{t}$ tend to infinity, which we write " $N \rightarrow+\infty$ " in short. We proceed in two steps.

Step 1: $T \geqslant \lim \sup _{N \rightarrow \infty} T_{N}$. We argue by contradiction. We denote by $u_{0}$ and $u_{1}$ the optimal controls for the continuous heat equation (under state constraints) in minimal time $T$. We then plug a suitable time-discretization of these controls into the discrete system. Using convergence results of the solutions of the discrete equation towards the continuous one (using the weak solutions in the sense of transposition as described above), we deduce that the corresponding discrete solutions, other than satisfying the state constraints, satisfy the convergence property $y_{N}(T) \rightarrow y(T)$ in $H^{-s}(0,1)$ for $s>3 / 2$ as $N \rightarrow+\infty$. This means that the discretizations of the continuous controls are quasi-controls for the discrete models. Now, since these discrete systems are uniformly controllable (see [3, 11, 27] for the analysis of uniform controllability of discrete versions of the $1 \mathrm{D}$ heat equation) and that the gap of the discrete final states $y_{N}(T)$ that we reach to the target tends to zero, in any time-interval of length $\delta$ with $\delta>0$ arbitrarily small, the discrete systems can be driven from $t=T$ to the target in time $t=T+\delta$ by means of controls whose size tends to zero and, therefore, so that the state preserve the nonnegativity constraint. This means that $T_{N} \leqslant T+\delta$ for all $\delta>0$ and $N$ large enough.

Step 2: $T \leqslant \liminf _{N \rightarrow \infty} T_{N}$. Let us denote by $T_{-}=\liminf _{N \rightarrow \infty} T_{N}$ and by $u_{0, N}$ and $u_{1, N}$ the corresponding discrete controls in the optimal times $T_{N}$. In view of the uniform controllability properties of these discrete models the controls should be bounded in the space of measures as $N \rightarrow+\infty$ (this specific aspect would require a finer study). Extracting subsequences, and passing weakly to the limit in the sense of measures, this would lead to limit controls $u_{0}$ and $u_{1}$ so that the corresponding solution of the continuous heat equation fulfils the nonnegativity constraint and reaches the target in time $T_{-}$. In case $T_{-}<T$, this would contradict the definition of $T$ being the minimal control time for the continuous model.

Remark 24. We have developed the numerical experiments in the $1 \mathrm{D}$ setting. Similar questions could be formulated in the multi-dimensional one. In that case the theory of uniform (with respect to the mesh size) controllability of numerical approximation schemes of the heat equation is much more complex. This is mainly due to the fact that Carleman inequalities are the main tool to prove the needed observability inequalities for the continuous heat equation. The extension of these inequalities to the discrete setting yields added high frequency remainder terms that require high frequency filtering to be used to ensure the convergence of numerical controls. In other words, the controllability property of the discrete dynamics has to be relaxed to deal only with the control of a low dimensional projection (see [2]). A systematic numerical investigation of the constrained control problem in the multi-dimensional case remains to be done.

\section{Conclusion, open problems and perspectives}

In this paper, we have addressed the problem of investigating controllability to steady-states for heat equations under nonnegativity state or control constraints. We have shown that, although controllability can be realized in arbitrarily small time when one does not take into account any constraint, such unilateral state or control constraints create a positive minimal time for realizing controllability.

Given an initial datum $\mathrm{y}^{0}$ and a final steady-state target $\mathrm{y}^{1}$, we have proved that $\underline{T}\left(\mathrm{y}^{0}, \mathrm{y}^{1}\right)>$ 0 (positive minimal time) in the following cases:

- 1D heat equation with Dirichlet boundary controls, under nonnegativity state or control constraints (both are equivalent); and in 1D, we have established more precise results: controllability with controls that are Radon measures at time $T=\underline{T}\left(\mathrm{y}^{0}, \mathrm{y}^{1}\right)$, lower estimates for $\underline{T}\left(\mathrm{y}^{0}, \mathrm{y}^{1}\right)$. 
- 1D heat equation with Neumann controls, under nonnegativity state constraint; lower estimate for $\underline{T}\left(\mathrm{y}^{0}, \mathrm{y}^{1}\right)$.

- Multi-D heat equation with Dirichlet boundary controls along the whole boundary, under nonnegativity state or control constraints (both are equivalent); controllability exactly in time $\underline{T}\left(\mathrm{y}^{0}, \mathrm{y}^{1}\right)$ with a Radon measure control; lower estimate for $\underline{T}\left(\mathrm{y}^{0}, \mathrm{y}^{1}\right)$ when the domain is a ball.

- Multi-D heat equation with Neumann boundary controls along the whole boundary, under nonnegativity state constraints; in contrast, under nonnegativity control constraints we have $\underline{T}\left(\mathrm{y}^{0}, \mathrm{y}^{1}\right)=+\infty$ (i.e., controllability fails: we have established it in 1D).

- Multi-D heat equation with internal control and/or Neumann boundary controls, under nonnegativity state constraints (existence of a Radon measure control in time $\underline{T}\left(\mathrm{y}^{0}, \mathrm{y}^{1}\right)$ is open).

The techniques presented in this paper can certainly be extended to some other related problems, among which:

- 1D parabolic equations with variable coefficients of the form $\partial_{t} y=\partial_{x}\left(a(x) \partial_{x} y\right)-p(x) \partial_{x} y$, with internal and/or boundary control (provided that the internal control is not acting everywhere). The results that we have established for the constant coefficient $1 \mathrm{D}$ heat equation can very likely be extended to this more general situation, by using spectral expansions and asymptotic properties of the spectrum of Sturm-Liouville problems, under suitable regularity assumptions on the coefficients.

- Finite-dimensional systems allowing for similar comparison properties and spectral expansions. More generally, in [15] we develop some specific finite-dimensional methods, exploiting the Brunovsky canonical form of controllable systems, allowing to deal with various constraints in the controlled state.

We present hereafter a non-exhaustive list of open problems and perspectives.

Uniqueness of controls at the minimal time. As observed above, if $y^{0} \in L^{2}(0,1)$ is symmetric with respect to $\frac{1}{2}$, then we can take $u_{0}=u_{1}$ (at least, if $T$ is large enough for them to exist). Besides, we have observed that the controls that we get with numerical simulations always coincide. It is thus natural to address the question of uniqueness of controls at the minimal time.

Let $\underline{T}$ be the minimal control time and let $\underline{u}_{0}$ and $\underline{u}_{1} \in \mathcal{M}(0, \underline{T})$ be nonnegative controls. Setting $\underline{u}=\frac{\underline{u}_{0}+\underline{u}_{1}}{2}$ and $\underline{h}=\frac{\underline{u}_{0}-\underline{u}_{1}}{2}$, we have, using (8),

$$
\begin{aligned}
& \int_{[0, \underline{T}]} e^{(2 p)^{2} \pi^{2} t} \mathrm{~d} \underline{h}(t)=0 \quad\left(p \in \mathbb{N}^{*}\right) \quad \text { and } \\
& \frac{\mathrm{y}^{1}}{(2 p+1)^{2} \pi^{2}}-\frac{e^{-(2 p+1)^{2} \pi^{2} \underline{T}} \mathrm{y}_{2 p+1}^{0}}{2(2 p+1) \pi}=\int_{[0, \underline{T}]} e^{-(2 p+1)^{2} \pi^{2}(\underline{T}-t)} \mathrm{d} \underline{u}(t) \quad(p \in \mathbb{N}) .
\end{aligned}
$$

These two equations characterize all possible controls $\underline{u}_{0}=\underline{u} \pm \underline{h}$ and $\underline{u}_{1}=\underline{u} \mp \underline{h} \in \mathcal{M}(0, \underline{T})$ in time $T$.

In order to prove that there is a unique pair of controls in minimal time, both components being the same, one needs to show that if $\underline{u}$ and $\underline{h}$ solve (33) and $\underline{u}+\underline{h}$ and $\underline{u}-\underline{h}$ are nonnegative, then $\underline{h}=0$.

Obviously, if $\underline{h} \neq 0$, then it has to change sign. On the other hand, if $\underline{u}$ and $\underline{h}$ are solutions of (33) and $\underline{u}+\underline{h}$ and $\underline{u}-\underline{h}$ are nonnegative, then we must have $\operatorname{supp} \underline{h} \subset \operatorname{supp} \underline{u}$.

With these observations in mind, nonuniqueness of the pair of controls might be expected to be true. Indeed, let $\underline{u}$ be nonnegative and satisfy the moment equations above. Let $\left[\tau_{1}, \tau_{2}\right]$ be a subinterval along which $\underline{u} \geqslant \delta>0$ for some $\delta>0$. Now, any function $\underline{h}$, supported on $\left[\tau_{1}, \tau_{2}\right]$ and satisfying the orthogonality conditions in (33), must change its sign as mentioned above. 
Such a function exists because the set of real exponentials involved in these moment equations is not complete in $L^{2}\left(\tau_{1}, \tau_{2}\right)$. Furthermore, $\underline{h}$ can be taken arbitrarily small in $L^{\infty}\left(\tau_{1}, \tau_{2}\right)$ norm. In these conditions, the new pair $(\underline{u}+\underline{h}, \underline{u}-\underline{h})$ satisfies (33) and is nonnegative. This would complete the argument for proving nonuniqueness, but to make it completely rigorous, we would need to show the existence of some subinterval $\left[\tau_{1}, \tau_{2}\right]$ along which $\underline{u} \geqslant \delta>0$. The argument above would fail if the interior of the support of the control $\underline{u}$ was empty. But we did not find computational evidence of this possible lack of uniqueness.

This issue would require a finer study of the optimality system characterizing the optimal controls at the minimal time.

Regularity of controls at the minimal time. Our analysis shows that the controls in minimal time can be guaranteed to be nonnegative Radon measures. Whether they actually belong to some smaller space (such as $L^{1}$ ) or really contain some nontrivial singular components is an open problem. This issue is related with the problem above on uniqueness of controls and their symmetry. Indeed, if controls were equal to Dirac deltas concentrated on some strategic times instances, then the argument above, which uses the fact that controls remain bounded away from zero on some open subintervals, would fail.

Turnpike structure. As mentioned above, the optimal pair that we obtain in the numerical experiments seem to present a turnpike and sparse structure with long lags along which the controls are identically equal to zero. A complete understanding of these properties for the continuous problem requires a finer analysis of the optimality system (Pontryagin maximum principle).

Convergence result for the minimal time. A complete analysis of the convergence of the minimal time for the discrete models towards the continuous one requires significant further work.

Sharpness of lower bounds. The numerical experiments indicate that the lower bounds that we have given for the minimal time are not sharp. Improving analytically these bounds is an open problem.

Numerical approximation in multi-D. A systematic analysis of the numerical approximation issues of constrained control in the multi-dimensional setting is to be developed.

Multi-D heat equations with variable coefficients. The arguments we have used to deal with the multi-D heat equation rely on the use of the radially symmetric eigenfunctions in the case where $\Omega$ is a ball, and on the use of comparison arguments. This method cannot be extended to general multi-D heat equations with variable coefficients which would require a separate treatment, certainly with different methods.

Nonlinear heat equations. Finally, similar questions arise naturally for nonlinear heat equations, and in particular semilinear ones, $\partial_{t} y=\Delta y+f(y)$. The arguments developed in this paper are based on spectral expansions and therefore are of a linear nature. Addressing nonlinearities is therefore a completely open challenge.

Acknowledgment. The authors thank Marius Tucsnak for pointing out towards relationships between the constrained control of the heat equation and the viscous Hamilton-Jacobi equation.

This work was partially supported by the Advanced Grant DYCON (Dynamic Control) of the European Research Council Executive Agency, ICON of the French ANR, FA9550-15-10027 of AFOSR, A9550-14-1-0214 of the EOARD-AFOSR, and the MTM2014-52347 Grant of the MINECO (Spain). 


\section{References}

[1] J. T. Betts. Practical methods for optimal control and estimation using nonlinear programming. 2nd ed. Philadelphia, PA: Society for Industrial and Applied Mathematics (SIAM), 2nd ed. edition, 2010.

[2] F. Boyer. On the penalised HUM approach and its applications to the numerical approximation of null-controls for parabolic problems. ESAIM, Proc., 41:15-58, 2013.

[3] F. Boyer, F. Hubert, and J. Le Rousseau. Uniform controllability properties for space/time-discretized parabolic equations. Numer. Math., 118(4):601-661, 2011.

[4] E. Casas and E. Zuazua. Spike controls for elliptic and parabolic PDEs. Syst. Control Lett., 62(4):311-318, 2013.

[5] J.-M. Coron. Control and nonlinearity. Providence, RI: American Mathematical Society (AMS), 2007.

[6] J.-M. Coron and E. Trélat. Global steady-state controllability of one-dimensional semilinear heat equations. SIAM J. Control Optim., 43(2):549-569, 2004.

[7] O. Y. Èmanuilov. Controllability of parabolic equations. Mat. Sb., 186(6):109-132, 1995.

[8] H. Fattorini and D. Russell. Exact controllability theorems for linear parabolic equations in one space dimension. Arch. Ration. Mech. Anal., 43:272-292, 1971.

[9] R. Fourer, D. M. Gay, and B. W. Kernighan. A modeling language for mathematical programming. Manage. Sci., 36(5):519-554, 1990

[10] M. Gugat, E. Trélat, and E. Zuazua. Optimal Neumann control for the 1D wave equation: finite horizon, infinite horizon, boundary tracking terms and the turnpike property. Syst. Control Lett., 90:61-70, 2016.

[11] S. Labbé and E. Trélat. Uniform controllability of semidiscrete approximations of parabolic control systems. Systems Control Lett., 55(7):597-609, 2006.

[12] G. Lebeau and L. Robbiano. Contrôle exact de l'équation de la chaleur. Comm. Partial Differential Equations, 20(1-2):335-356, 1995.

[13] J. Lions and E. Magenes. Problèmes aux limites non homogenes et applications. Vol. 1, 2. Paris: Dunod 1: XIX, 372 p.; 2: XV, 251 p. (1968)., 1968.

[14] J.-L. Lions. Contrôlabilité exacte, perturbations et stabilisation de systèmes distribués. Tome 1, volume 8 of Recherches en Mathématiques Appliquées [Research in Applied Mathematics]. Masson, Paris, 1988. Contrôlabilité exacte. [Exact controllability], With appendices by E. Zuazua, C. Bardos, G. Lebeau and J. Rauch.

[15] J. Lohéac, E. Trélat, and E. Zuazua. Minimal controllability time for linear finite dimensional systems with state constraints. In preparation.

[16] A. Münch and E. Zuazua. Numerical approximation of null controls for the heat equation: ill-posedness and remedies. Inverse Probl., 26(8):39, 2010.

[17] A. Porretta and E. Zuazua. Null controllability of viscous Hamilton-Jacobi equations. Ann. Inst. Henri Poincaré, Anal. Non Linéaire, 29(3):301-333, 2012.

[18] E. Schmidt. Boundary control for the heat equation with steady-state targets. SIAM J. Control Optim., 18:145-154, 1980.

[19] T. I. Seidman. Observation and prediction for the heat equation. III. J. Differential Equations, 20(1):18-27, 1976.

[20] E. Trélat. Contrôle optimal. Théorie et applications. Paris: Vuibert, 2005.

[21] E. Trélat. Optimal control and applications to aerospace: some results and challenges. $J$. Optim. Theory Appl., 154(3):713-758, 2012.

[22] E. Trélat, C. Zhang, and E. Zuazua. Steady-state and periodic exponential turnpike property for optimal control problems in Hilbert spaces. Preprint Hal, Oct. 2016. 
[23] E. Trélat and E. Zuazua. The turnpike property in finite-dimensional nonlinear optimal control. J. Differ. Equations, 258(1):81-114, 2015.

[24] M. Tucsnak. Controllability with positivity type constraints: local and global results. LIASFMA Autumn School and Workshop on Control and Inverse Problems for Partial Differential Equations, Zhejiang University Hangzhou, China, Oct 2016.

[25] M. Tucsnak and G. Weiss. Observation and control for operator semigroups. Basel: Birkhäuser, 2009.

[26] A. Wächter and L. T. Biegler. On the implementation of an interior-point filter linesearch algorithm for large-scale nonlinear programming. Math. Program., 106(1 (A)):25$57,2006$.

[27] E. Zuazua. Control and numerical approximation of the wave and heat equations. In Proceedings of the international congress of mathematicians (ICM), Madrid, Spain, August 22-30, 2006. Volume III: Invited lectures, pages 1389-1417. Zürich: European Mathematical Society (EMS), 2006.

[28] E. Zuazua. Controllability and observability of partial differential equations: some results and open problems. In Handbook of differential equations: Evolutionary equations. Vol. III, pages 527-621. Amsterdam: Elsevier/North-Holland, 2007. 